\title{
Multiparametric profiling of non-small-cell lung cancers reveals distinct immunophenotypes
}

Patrick H. Lizotte, ${ }^{1,2}$ Elena V. Ivanova, ,, Mark M. Awad, ${ }^{2,3,4}$ Robert E. Jones, ${ }^{1,2}$ Lauren Keogh, ${ }^{1,2}$ Hongye Liu, ${ }^{1,2}$ Ruben Dries, ${ }^{2,3,5}$ Christina Almonte, ${ }^{2}$ Grit S. Herter-Sprie, ${ }^{2}$ Abigail Santos, ${ }^{2,3}$ Nora B. Feeney, ${ }^{1,2}$ Cloud P. Paweletz,, ${ }^{1,2}$ Meghana M. Kulkarni,, ${ }^{1,2}$ Adam J. Bass, ${ }^{2,4}$ Anil K. Rustgi, ${ }^{6}$ Guo-Cheng Yuan,, Donald W. Kufe, , ${ }^{2,4}$ Pasi A. Jänne, ${ }^{1,2,3,4}$ Peter S. Hammerman, ${ }^{2,3,4}$ Lynette M. Sholl, ${ }^{4,8}$ F. Stephen Hodi, ${ }^{2,4}$ William G. Richards, ${ }^{4,9,10}$ Raphael Bueno, ${ }^{4,9,10}$ Jessie M. English, ${ }^{1,2}$ Mark A. Bittinger, ${ }^{1,2}$ and Kwok-Kin Wong ${ }^{1,2,3,4}$

'Belfer Center for Applied Cancer Science, Boston, Massachusetts, USA. ${ }^{2}$ Department of Medical Oncology and ${ }^{3}$ Lowe Center for Thoracic Oncology, Dana-Farber Cancer Institute, Boston, Massachusetts, USA. ${ }^{4}$ Harvard Medical School, Boston, Massachusetts, USA. ${ }^{5}$ Department of Biostatistics and Computational Biology, Dana-Farber Cancer Institute, Boston, Massachusetts, USA. ${ }^{6}$ Division of Gastroenterology, Departments of Medicine and Genetics, Abramson Cancer Center, Perelman School of Medicine, University of Pennsylvania, Philadelphia, Pennsylvania, USA ${ }^{7}$ Harvard Chan School of Public Health, Boston, Massachusetts, USA. ${ }^{8}$ Department of Pathology, ${ }^{9}$ Division of Thoracic Surgery, and ${ }^{10}$ International Mesothelioma Program of the Lung Center Surgery, Brigham and Women's Hospital, Boston, Massachusetts, USA.

BACKGROUND. Immune checkpoint blockade improves survival in a subset of patients with non-small-cell lung cancer (NSCLC), but robust biomarkers that predict response to PD-1 pathway inhibitors are lacking. Furthermore, our understanding of the diversity of the NSCLC tumor immune microenvironment remains limited.

METHODS. We performed comprehensive flow cytometric immunoprofiling on both tumor and immune cells from 51 NSCLCs and integrated this analysis with clinical and histopathologic characteristics, next-generation sequencing, mRNA expression, and PD-L1 immunohistochemistry (IHC).

RESULTS. Cytometric profiling identified an immunologically "hot" cluster with abundant CD8 ${ }^{+} T$ cells expressing high levels of PD- 1 and TIM- 3 and an immunologically "cold" cluster with lower relative abundance of $\mathrm{CD8^{+ }} T$ cells and expression of inhibitory markers. The "hot" cluster was highly enriched for expression of genes associated with $\mathrm{T}$ cell trafficking and cytotoxic function and high PD-L1 expression by IHC. There was no correlation between immunophenotype and KRAS or EGFR mutation, or patient smoking history, but we did observe an enrichment of squamous subtype and tumors with higher mutation burden in the "hot" cluster. Additionally, approximately $20 \%$ of cases had high B cell infiltrates with a subset producing IL-10.

Authorship note: P.H. Lizotte and E.V. Ivanova contributed equally to this work.

Conflict of interest: The authors have declared that no conflict of interest exists.

Submitted: June 8, 2016 Accepted: August 4, 2016 Published: September 8, 2016

Reference information: JCI Insight. 2016;1(14):e89014. doi:10.1172/jci.insight.89014.
CONCLUSIONS. Our results support the use of immune-based metrics to study response and resistance to immunotherapy in lung cancer.

FUNDING. The Robert A. and Renée E. Belfer Family Foundation, Expect Miracles Foundation, Starr Cancer Consortium, Stand Up to Cancer Foundation, Conquer Cancer Foundation, International Association for the Study of Lung Cancer, National Cancer Institute (R01 CA205150), and the Damon Runyon Cancer Research Foundation. 


\section{Introduction}

The development of therapies that block inhibitory receptors expressed by $\mathrm{T}$ lymphocytes has revolutionized cancer treatment. The Food and Drug Administration approved the use of the PD-1 inhibitor nivolumab for treatment of advanced squamous non-small-cell lung cancer (NSCLC) in March of 2015 $(1,2)$; this approval was later extended to nonsquamous NSCLC in October of that same year, the same month that the PD-1 inhibitor pembrolizumab was granted accelerated approval for treatment of advanced NSCLC expressing the PD-1 ligand PD-L1 (3). Approval of both agents for NSCLC constituted a watershed moment for immunotherapy and also for the treatment of lung cancer, which is the second most common cancer type and the leading cause of cancer death in the United States (4). There are currently over 100 ongoing clinical trials involving PD-1/PD-L1 pathway blockade in NSCLC.

While clinical responses to immunomodulatory agents have been impressive, the field has been striving to better understand response and resistance to improve patient selection and to aid in the design of rational combination therapy approaches. Objective response rates to nivolumab treatment of 33\% (2), 15\% (5), and $20 \%$ (6) have been reported for squamous NSCLC; and rates of $12 \%(2), 17 \%(7)$, and 19\% (1) for have been reported for nonsquamous NSCLC. Comparable response rates of $19.4 \%$ (3) and $23 \%$ (8) have been reported for the PD-1 inhibitor pembrolizumab and the PD-L1 inhibitor atezolizumab, respectively, for either histological subtype. Higher objective response rates have been observed in NSCLC patients with PD-L1 ${ }^{+}$ tumors, as assessed by immunohistochemistry $(\mathrm{IHC})(3,8)$, and, in particular, responses were highest in patients with PD-L1+ immune cells (8). However, PD-L1 IHC has limitations as a diagnostic; the response rates are generally higher in $\mathrm{PD}-\mathrm{L}^{+}$tumors but approach a maximum of $39 \%$ (9) or $45 \%$ (3) in tumors with $>50 \%$ PD-L1 positivity and some PD-L1 ${ }^{-}$tumors also respond to therapy. The methods to assay and interpret PD-L1 IHC are both diverse and subjective and require further validation, as early results from the BluePrint PD-L1 Assay Harmonization Study have shown (10).

The immune microenvironment is complex, dynamic, and spatially heterogeneous. There are numerous immunosuppressive mechanisms in addition to the PD-1/PD-L1 axis, which may explain why an immunological metric such as PD-L1 IHC positivity is predictive of response to anti-PD-1 therapy in less than half of patients. T cells are capable of expressing multiple inhibitory receptors concurrently, and this compensatory upregulation may account for resistance to PD-1 blockade. For instance, it has recently been demonstrated that the alternative immune checkpoint TIM-3 is upregulated by T cells engaged by anti-PD-1, and this may explain adaptive resistance to anti-PD-1 therapy (11). Response to checkpoint blockade is also likely affected by cytotoxic T lymphocyte-extrinsic (CTL-extrinsic) factors as well, such as the presence of myeloid-derived suppressor cells (MDSCs) and FOXP3 ${ }^{+}$Tregs, the latter of which have been documented in NSCLC (12). The presence of MDSCs and Tregs in NSCLC is positively correlated with an abundance of IL-10-producing B regulatory cells (Bregs); all three are associated with NSCLC progression (13).

Response to anti-PD-1 therapy in NSCLC may also be affected by traditional stratifying criteria, such as histological subtype, specific oncogenic driver mutations, and smoking history. For instance, low PD-1 expression in the tumor is correlated with KRAS mutation and low PD-L1 expression is correlated with the presence of EGFR mutations (14). Activating EGFR mutations and adenocarcinomas are more commonly found in never-to-light smokers as compared with smokers, whereas there is a stronger association between smoking and the squamous subtype (15). Smoking is known to increase the prevalence of somatic mutations. Higher mutation rates have been shown to increase the probability that tumors display neoantigens that can trigger T cell-mediated tumor cell lysis and, by extension, enhance response to checkpoint blockade (16).

It is important to understand the immunological landscape of NSCLC, as this information might reveal mechanisms of response and resistance to specific immunomodulatory agents and inform future development of more effective combination approaches. Comprehensive immunoprofiling may enable identification of robust metrics for determining candidacy to receive specific immunomodulatory therapies, and some approaches, including multiplexed slide-based analysis (17) and gene expression profiling (18), have been used to define components of the NSCLC immune microenvironment, with the goal of developing more robust biomarkers beyond PD-L1 IHC. To this end, we performed in-depth immunoprofiling by flow cytometry and focused mRNA profiling of immuno-oncology relevant genes and used a multidimensional cluster algorithm $(19,20)$ to determine if distinct immunological subtypes exist in NSCLC and whether these features correlate with mutation status, histological subtype, PD-L1 IHC, and smoking history. Our studies identified heterogeneity in the NSCLC immune microenvironment and suggest that different immunotherapy approaches may be needed for specific subsets of NSCLC patients. 


\section{Table 1. NSCLC sample characteristics}

\begin{tabular}{|c|c|}
\hline Sample characteristic & No. (\%) \\
\hline Total NSCLC cases & $51(100 \%)$ \\
\hline \multicolumn{2}{|l|}{ Sex } \\
\hline Male & $21(41 \%)$ \\
\hline Female & $29(57 \%)$ \\
\hline \multicolumn{2}{|l|}{ Race } \\
\hline European descent & 43 (84\%) \\
\hline Asian & $2(4 \%)$ \\
\hline African-American & $3(6 \%)$ \\
\hline Unknown & $3(6 \%)$ \\
\hline \multicolumn{2}{|l|}{ Histology } \\
\hline Adenocarcinoma & $42(82 \%)$ \\
\hline Squamous & $7(14 \%)$ \\
\hline Adenosquamous & $1(2 \%)$ \\
\hline PD-L1 immunohistochemistry completed & $43(84 \%)$ \\
\hline Tumor cell ${ }^{+} /$immune cell ${ }^{+}$ & $7(14 \%)$ \\
\hline Tumor cell+/immune cell- & $2(4 \%)$ \\
\hline Tumor cell-/immune cell ${ }^{+}$ & $5(10 \%)$ \\
\hline Tumor cell-/immune cell- & $29(57 \%)$ \\
\hline $\begin{array}{l}\text { Next-generation sequencing or ddPCR } \\
\text { completed }\end{array}$ & $50(98 \%)$ \\
\hline KRAS mutation & $18(35 \%)$ \\
\hline EGFR mutation & $7(14 \%)$ \\
\hline Nanostring expression analysis completed & $29(57 \%)$ \\
\hline \multicolumn{2}{|l|}{ Neoadjuvant chemotherapy } \\
\hline Treated with neoadjuvant chemotherapy & $7(14 \%)$ \\
\hline Did not receive neoadjuvant chemotherapy & $42(82 \%)$ \\
\hline \multicolumn{2}{|l|}{ Stage ( $\mathrm{A}$ and $\mathrm{B})$} \\
\hline I & 23 (45\%) \\
\hline II & $10(20 \%)$ \\
\hline III & $9(18 \%)$ \\
\hline IV & $7(14 \%)$ \\
\hline \multicolumn{2}{|l|}{ Smoking status } \\
\hline$>30$ pack years & 19 (37\%) \\
\hline$\leq 30$ pack years & 20 (39\%) \\
\hline Current smoker & $6(12 \%)$ \\
\hline Former smoker & 33 (65\%) \\
\hline Never smoker & $11(22 \%)$ \\
\hline
\end{tabular}

\section{Results}

Characteristics of the NSCLC data set. We compiled a data set with clinical annotation (Table 1) of 51 freshly resected NSCLC samples, including immunohistochemical determination of PD-L1 status, next-generation sequencing $(21,22)$, digital droplet PCR for oncogenic driver mutations, mRNA expression analysis by Nanostring, and comprehensive immunoprofiling of dissociated bulk resected tumors by multiparametric flow cytometry. As part of our effort to comprehensively characterize the tumor immune microenvironment of NSCLC, we utilized multiple antibody panels (Supplemental Figure 1; supplemental material available online with this article; doi:10.1172/jci.insight.89014DS1) to determine the abundance of major immune cell lineages. We focused on the characterization of $\mathrm{CD} 4^{+}$ and $\mathrm{CD}^{+} \mathrm{T}$ cells, including their differentiation status (FOXP3, CCR7, CD45RA, CD45RO), expression of activation markers (CD69, CD11a, CD38) and inhibitory receptors (PD-1, TIM-3, LAG-3, CTLA-4), and their proliferation (Ki-67). We also used flow cytometry to measure PD-L1 and PD-L2 expression on monocytes, granulocytes, and tumor cells (defined as $\mathrm{CD}^{-}$EpCAM $^{+}$). Clinical, pathologic, and genomic data are shown for each sample, along with proportions of T cells, B cells, granulocytes, monocytes, NK cells, and NT T cells (Figure 1). There was considerable variability in the proportion of immune cells that were $\mathrm{CD}^{+} \mathrm{T}$ cells, from $1.4 \%$ to $44.6 \%$ (mean $11.50 \% \pm 1.476 \%$, SEM, Figure 1). Tumors also exhibited markedly heterogeneity in total leukocyte infiltrate, with $\mathrm{CD} 45^{+}$cells comprising percentages as low as $16 \%$ or as high as $97.3 \%$ of live cells (mean $69.95 \% \pm$ $3.074 \%$, SEM). Similarly, we observed a high degree of heterogeneity in the immune infiltrate across this set of tumors.

We performed unsupervised hierarchical clustering on tumor and matched normal lung samples using comprehensive immunophenotypic data (Figure 2). As expected, normal lung tissue samples clustered together and tumor samples clustered together. We observed a relative absence in expression of inhibitory receptors by $\mathrm{CD}^{+}$and $\mathrm{CD} 8^{+} \mathrm{T}$ cells in normal lung as compared with tumors. It is notable that tumors clustering with normal lung displayed high granulocytic infiltrate, which is what we consistently observe in normal lung (Figure 2).

We first analyzed major leukocyte lineages based on clinical, genomic, and histopathologic criteria. We observed no significant difference in the immune cell subsets in tumors from patients who had never smoked as compared with patients with a history of tobacco use (Figure 3A). There was also no significant difference when immune infiltrates were compared by tumor histology, although squamous tumors displayed a slight enrichment $(P<0.05)$ in granulocytes as compared with adenocarcinoma (Figure 3B). Furthermore, there were no significant immune cell differences in KRAS mutant tumors or EGFR mutant tumors or tumors that did not harbor mutations in either of these genes (Figure 3C). Compared with normal lung, tumors consistently showed a significantly increased abundance of $\mathrm{CD} 19^{+} \mathrm{B}$ cells, FOXP3 ${ }^{+}$Tregs, $\mathrm{CD} 8^{+} \mathrm{T}$ cells, and particularly CD45RO ${ }^{+}$memory $\mathrm{CD} 8^{+} \mathrm{T}$ cells and a significant relative lack of NK cells and NK T cells (Figure $3 \mathrm{~B}$ ). We observed increased CD $8^{+}$ $\mathrm{T}$ cells and memory $\mathrm{CD}^{+} \mathrm{T}$ cells in $\mathrm{PD}-\mathrm{L} 1^{+}$tumors as compared with PD-L1- tumors (Figure 3D). There was no statistically significant difference in immune cell populations among samples that were $\mathrm{PD}-\mathrm{L} 1^{+}$in tumor cells $\left(\mathrm{TC}^{+} \mathrm{IC}^{+}\right.$and $\left.\mathrm{TC}^{+} \mathrm{IC}^{-}\right)$compared with those that were $\mathrm{PD}-\mathrm{L}^{+}$only in immune cells $\left(\mathrm{TC}^{-} \mathrm{IC}^{+}\right)$ (Figure 3D).

Due to the use of PD-1 inhibitors for the treatment of NSCLC and the known upregulation of additional checkpoint receptors that may be associated with resistance to PD-1 therapy (11), we analyzed the expression of PD-1, TIM-3, CTLA-4, and LAG-3 on CD4 ${ }^{+}$and CD8 ${ }^{+}$T cells. Smoking status did not significantly correlate with inhibitory receptor expression (Figure 4A and Supplemental Figure 2). There was significantly increased expression of inhibitory receptors by $\mathrm{CD} 4^{+} \mathrm{T}$ cells in squamous tumors rel- 

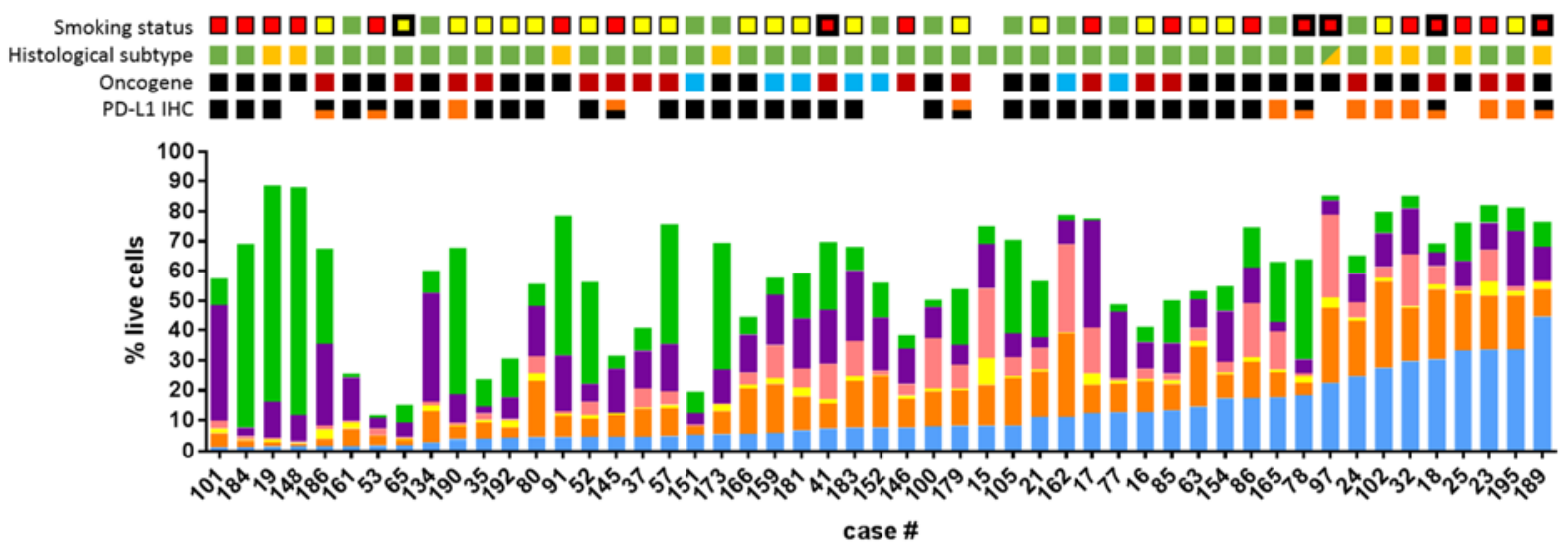
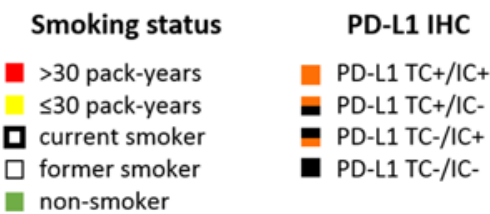
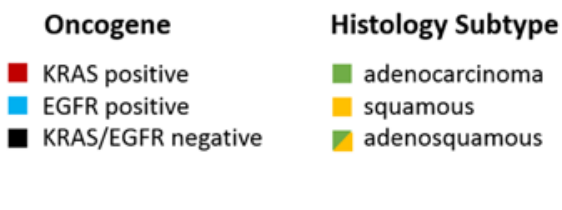

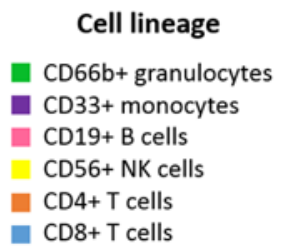

Cell lineage

Figure 1. Clinical characteristics of NSCLC data set. Major immune cell lineages profiled from 51 NSCLC patients are depicted as the percentage of live cells and arranged by increasing percentage of CD8 ${ }^{+} \mathrm{T}$ cells. Colored tile tracks above indicate smoking status, histological subtype, mutant KRAS or EGFR, and PD-L1 IHC.

ative to adenocarcinoma. For $\mathrm{CD}^{+} \mathrm{T}$ cells, we observed no significant difference in levels of PD-1 and TIM-3 in squamous tumors compared with adenocarcinoma, but we did observe significantly elevated CTLA-4 in squamous tumors. Squamous tumors also displayed significantly increased coexpression of TIM-3 and PD-1 in both CD8 ${ }^{+}$and $\mathrm{CD}^{+} \mathrm{T}$ cells (Supplemental Figure 2). Inhibitory receptor expression was uniformly higher in tumor samples when compared with normal lung (Figure 4B). In adenocarcinomas, KRAS-driven tumors exhibited significantly increased expression of PD-1 on both $\mathrm{CD} 4^{+}$and $\mathrm{CD}^{+} \mathrm{T}$ cells relative to EGFR-driven and KRAS/EGFR ${ }^{-}$tumors (Figure $4 \mathrm{C}$ ). Both $\mathrm{CD} 4^{+}$and $\mathrm{CD} 8^{+}$ $\mathrm{T}$ cells from PD-L1 ${ }^{+}$tumors displayed significant upregulation of individual inhibitory receptors and, similar to what we observed with our analysis by leukocyte lineage (Figure 3D), there were no significant differences in PD-1, TIM-3, and CTLA-4 expression between PD-L1 TC ${ }^{+}$and $\mathrm{TC}^{-} \mathrm{IC}^{+}$tumors (Figure 4D). $\mathrm{TC}^{+}$and $\mathrm{TC}^{-} \mathrm{IC}^{+}$tumors also had increased coexpression of TIM-3 and PD-1 on CD $4^{+}$and $\mathrm{CD} 8^{+} \mathrm{T}$ cells relative to $\mathrm{PD}-\mathrm{L}^{-}{ }^{-}$tumors (Supplemental Figure 2). LAG-3 was uniformly not expressed by $\mathrm{T}$ cells across our NSCLC cohort.

NSCLCS cluster into distinct immunophenotypes. With the expanding clinical application of immunomodulatory agents such as PD-1 blockade for treatment of NSCLC, knowledge of the immunophenotype of the tumor with a high degree of granularity may enable the development of robust biomarkers for patient selection. We employed the t-distributed stochastic neighbor embedding ( $\mathrm{t}$-SNE) multidimensional reduction algorithm $(19,23)$ to analyze our multiparametric immunophenotypic data in an unbiased manner. Close proximity individual NSCLC cases indicate immunophenotypic similarity. Tumor samples separated into two main groups, which we denoted as immunologically "hot" cluster 1 and immunologically "cold" cluster 2 , and a third small group defined by high granulocytic infiltrate (gran+) that we grouped within the "hot" cluster based on proximity and shared T cell phenotypic markers (Figure 5). The immunologically "hot" cluster was differentiated by the presence of inflammatory markers, including an abundance of $\mathrm{CD}^{+} \mathrm{T}$ cells and high expression of inhibitory receptors - particularly PD-1 and TIM-3 - on those CD8 ${ }^{+} \mathrm{T}$ cells (Figure $5, \mathrm{~A}-\mathrm{C}$ ). This cluster was further differentiated by infiltration of FOXP3 ${ }^{+}$Tregs and by expression of PD-L1 on tumor cells and immune cells (Figure 5, D-F). Tumor cell PD-L1 positivity by IHC is directly correlated with PD-L1 expression on EpCAM ${ }^{+}$tumors cells, as detected by flow cytometry (Supplemental Figure 3 ).

We also observed intracluster heterogeneity, with notable examples indicated by arrows and reference case numbers (Figure 5, A-C, E, and F). For example, case 78 displayed high $\mathrm{CD} 8^{+} \mathrm{T}$ cell infiltrate with high coexpression of PD-1 and TIM-3, compared with case 25 , which had high $\mathrm{CD} 8^{+} \mathrm{T}$ cell infiltrate with no expression of PD-1 or TIM-3. Case 23 had high PD-1 expression by CD8 ${ }^{+} \mathrm{T}$ cells and high PD-L1 


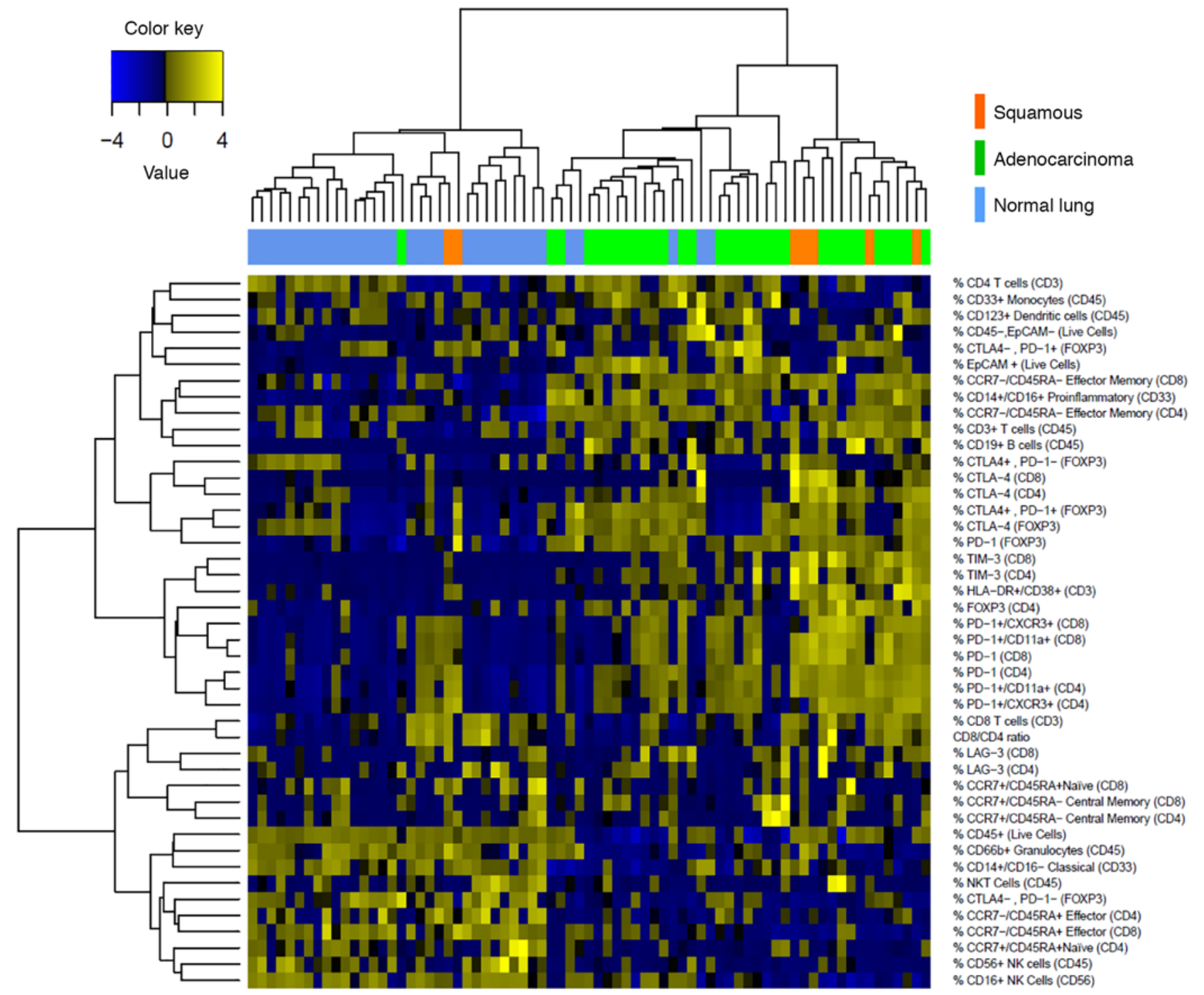

Figure 2. Clustering of NSCLC data set. Unbiased hierarchical clustering of 51 NSCLC samples and, where available, matched normal lung (top row). Immune parameters measured by multicolor flow cytometry are listed. Tiles are shaded by percentage of expression of markers.

staining on tumor cells but no TIM-3 on $\mathrm{CD}^{+} \mathrm{T}$ cells. We observed a highly significant positive correlation between PD-1 and TIM-3 expression by both $\mathrm{CD} 8^{+}$and $\mathrm{CD} 4^{+} \mathrm{T}$ cells (Supplemental Figure 4 ). There were no cases in which CD8 ${ }^{+} \mathrm{T}$ cells expressed TIM-3 and not PD-1, but in approximately $20 \%$ of cases CD ${ }^{+}$ T cells expressed PD-1 and not TIM-3. It is our hope that multicolor flow cytometry will be employed in future prospective studies of patients receiving immunotherapy and that the high level of immunophenotypic granularity generated by this methodology may help explain heterogeneity in response to anti-PD-1 therapy in NSCLCs and/or tumors with high degrees of lymphocytic infiltrates.

Adenocarcinomas were equally distributed between "hot" and "cold" clusters, whereas squamous tumors were enriched in the "hot" cluster (Figure 5G). Mutant KRAS and EGFR were not associated with immunophenotype, even though all 8 EGFR-mutated tumors were PD-L1- by IHC (Figure 5H). Heavy smokers ( $>30$ pack years) appeared concentrated in the "hot" cluster relative to smokers ( $<30$ pack years) and never smokers, but this was not statistically significant. We also analyzed smoking status based on pack years and found a trending correlation $(P=0.11)$ between immunophenotype and smoking status, with higher pack year smokers concentrated in the "hot" cluster (Figure 5I).

Forty-two of fifty-one of our cases were profiled by next-generation sequencing (21, 22). A forthcom- 

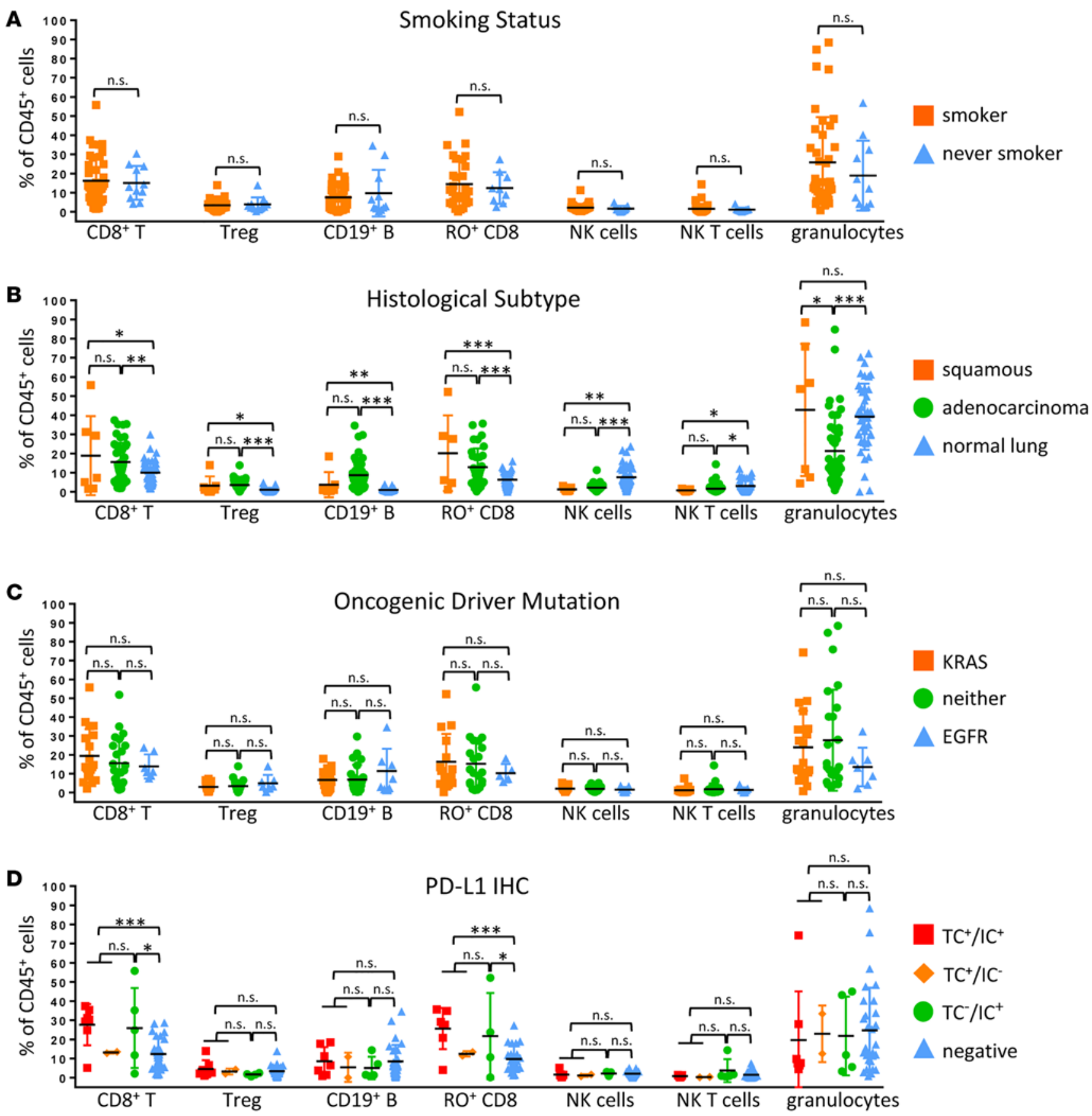

Figure 3. Immune cell lineages by clinical features. Percentages of total CD45+ cells based on smoking history (A), histological subtype (B), oncogene status (C), and PD-L1 immunohistochemical scoring (D) of tumor cells $\left(\mathrm{TC}^{+}\right)$and immune cells $\left(\mathrm{IC}^{+}\right)$of major immune cell lineages that vary significantly between tumors are presented. Data for bar graphs were calculated using unpaired Student's $t$ test. ${ }^{*} P<0.05 ;{ }^{* *} P<0.01 ;{ }^{* * *} P<0.001$. Mean with SD. Two-way ANOVA: smoker vs. never smoker, $P=0.4207$; adenocarcinoma vs. squamous, $P=0.0362$; adenocarcinoma vs. normal lung, $P=0.6034$; squamous vs. normal lung, $P=0.0332$; EGFR vs. KRAS, $P=0.1901$; KRAS vs. neither, $P=0.9915$; EGFR vs. neither, $P=0.2636 ; \mathrm{TC}^{+}$vs. IC ${ }^{+}, P=0.9990 ; \mathrm{TC}^{+}$vs. negative, $P=0.0630 ; \mathrm{IC}^{+}$vs. negative, $P=0.1667$.

ing publication shows that somatic mutational burden quantified from whole-exome sequencing and mutation burden extrapolated from this platform are well correlated (24). We, therefore, used the total number of mutations from our OncoPanel results as a proxy and found that mutational burden was significantly higher in tumors in the "hot" cluster (Figure 5J). Mutational burden also correlated with smoking status, as expected (Supplemental Figure 5).

Immunologically "hot" NSCLC tumors display unique phenotype. The unbiased t-SNE clustering was based 
A

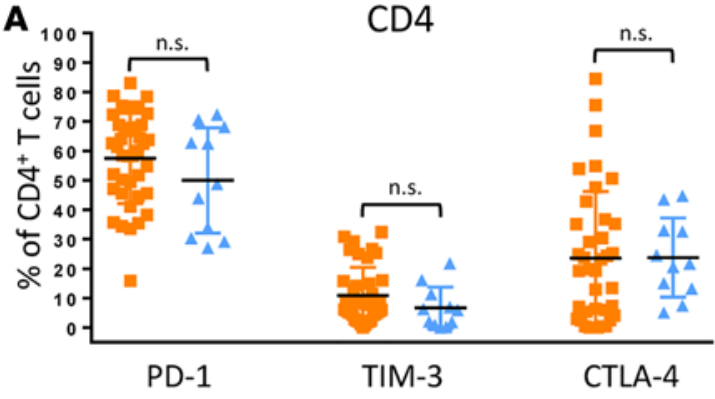

B

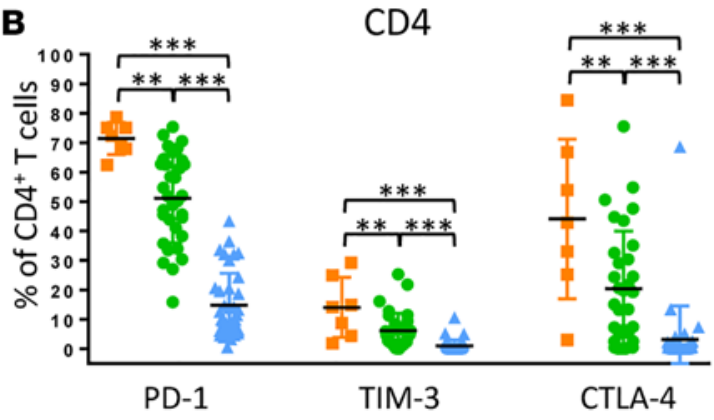

C
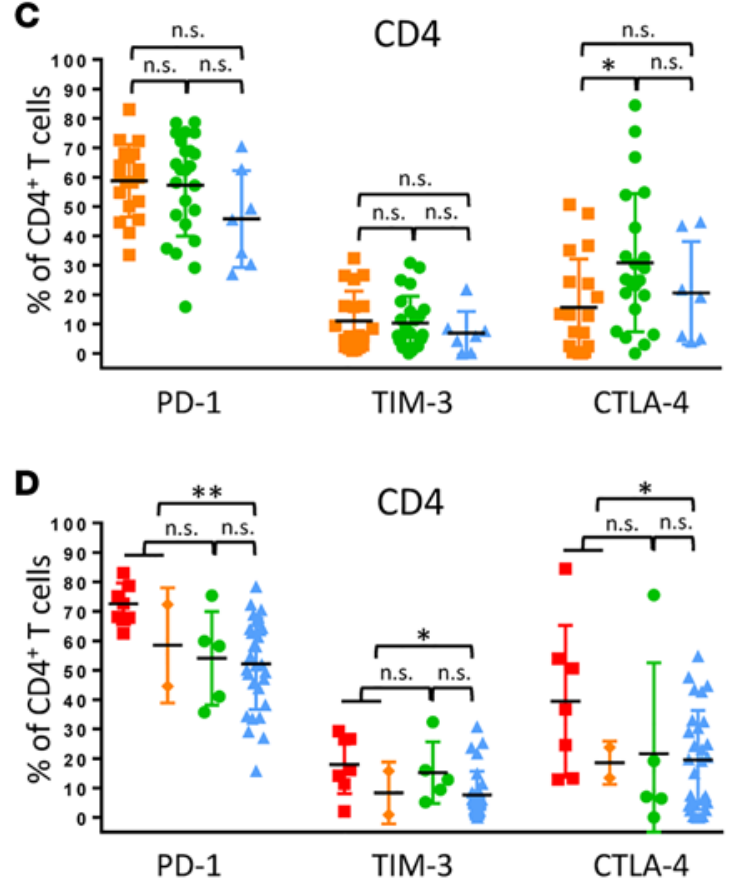

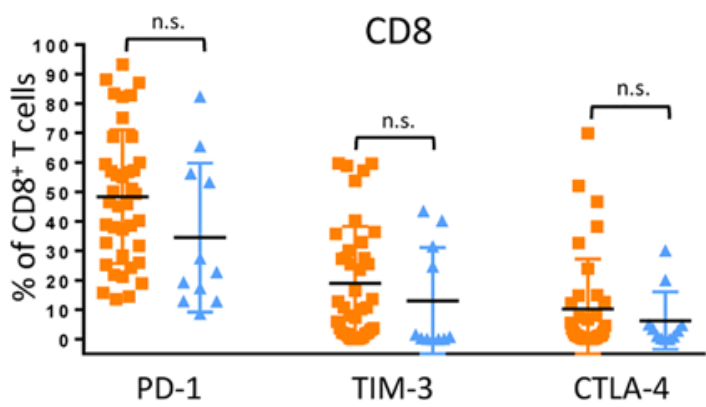

Smoking Status

smoker

never smoker

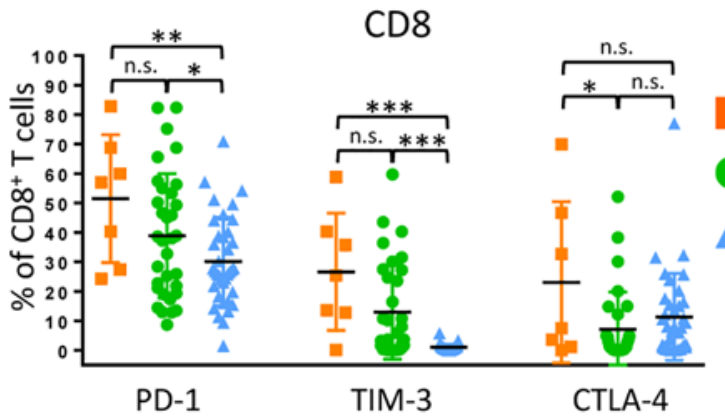

Subtype

squamous

adenocarcinoma

normal lung
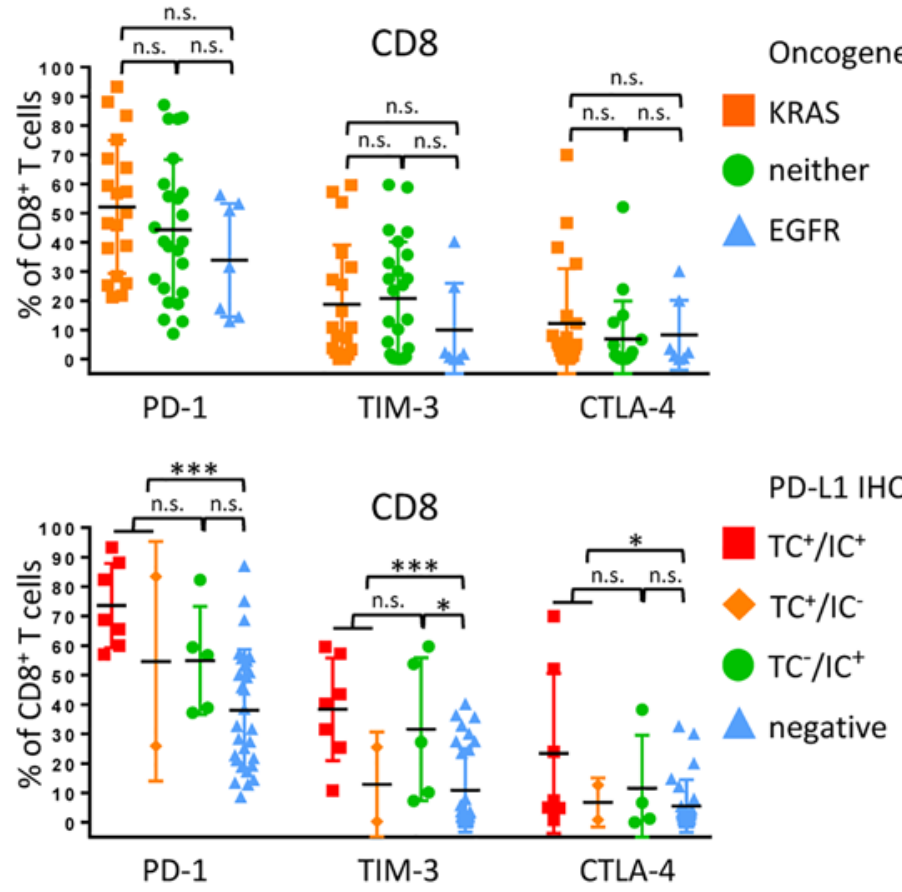

Figure 4. T cell expression of inhibitory receptors by clinical features. Percentage of expression of inhibitory receptors PD-1, TIM-3, and CTLA - 4 by CD4 T cells (left) and CD8+T cells (right) based on smoking history (A), histological subtype (B), oncogene status (C), and PD-L1 immunohistochemical scoring (D) of tumor cells $\left(\mathrm{TC}^{+}\right)$and immune cells $(\mathrm{IC}+)$. Data for bar graphs were calculated using unpaired Student's $t$ test with. ${ }^{*} P<0.05$; ${ }^{* *} P<0.01$; ${ }^{* * *} P<$ 0.001. Mean with SD. Two-way ANOVA CD4 ${ }^{+}$T cells: smoker vs. never smoker, $P=0.2314$; adenocarcinoma vs. squamous, $P<0.0001$; adenocarcinoma vs. normal lung, $P<0.0001$; squamous vs. normal lung, $P<0.0001$; EGFR vs. KRAS, $P=0.2450$; KRAS vs. neither, $P=0.1272$; EGFR vs. neither, $P=0.0619$; TC ${ }^{+}$ vs. IC $+P=0.1038$; TC + vs. negative, $P<0.001$; IC+ vs. negative, $P=0.3626$. Two-way ANOVA CD8 ${ }^{+}$T cells: smoker vs. never smoker, $P=0.0433$; adenocarcinoma vs. squamous, $P=0.0017$; adenocarcinoma vs. normal lung, $P=0.0083$; squamous vs. normal lung, $P<0.0001 ;$ EGFR vs. KRAS, $P=0.0759 ;$ KRAS vs. neither, $P=0.9649$; EGFR vs. neither, $P=0.0972 ; \mathrm{TC}^{+}$vs. $\mathrm{IC}^{+}, P=0.2742 ; \mathrm{TC}^{+}$vs. negative, $P<0.0001 ; \mathrm{IC}^{+}$vs. negative, $P=0.0025$.

on flow cytometric profiling, and, in order to augment our classification of immunologically "hot" and "cold" tumors, we performed a focused transcriptomic analysis using the Nanostring Human PanCancer Immune Profiling Panel (18). Our "hot" cluster showed significant enrichment in CXCL9, CXCL10, IDO1, granzyme B, IFN- $\gamma$, and STAT1, which aligns with the "IFN- $\gamma$ signature" reported elsewhere $(25$, 
A $\mathrm{CD} 8+$
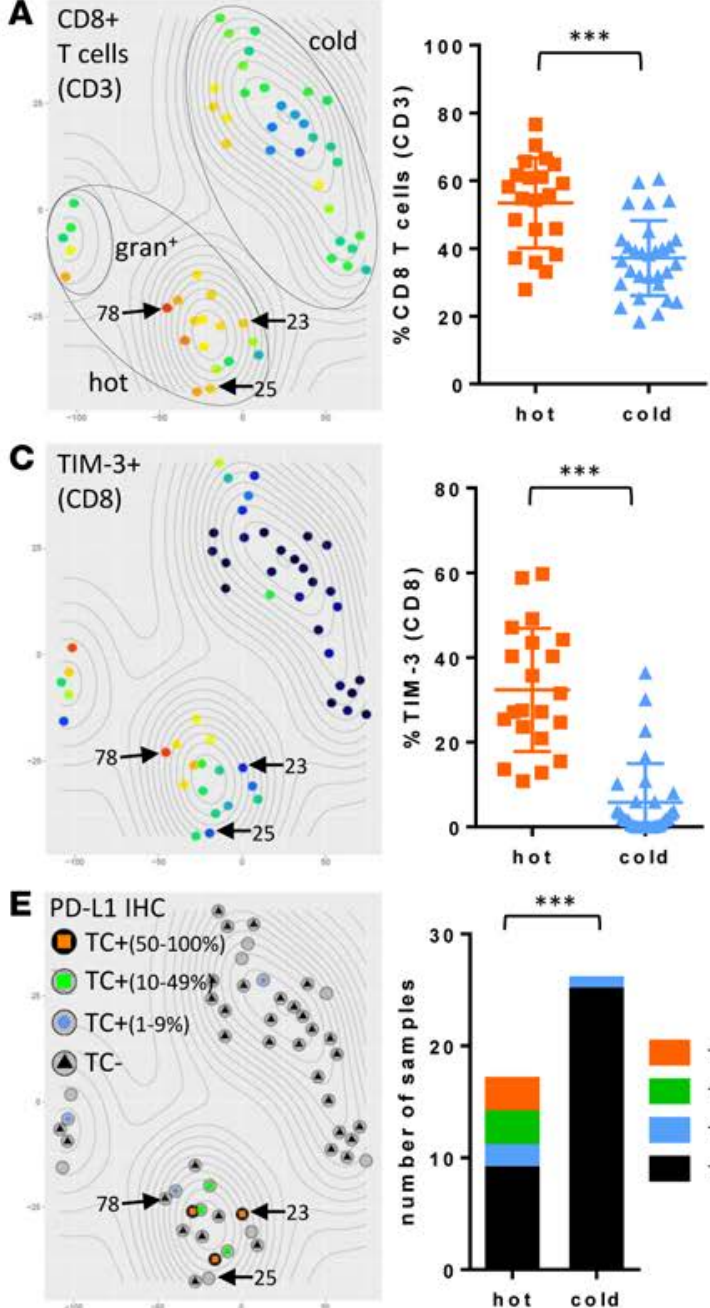

E PD-L1 IHC

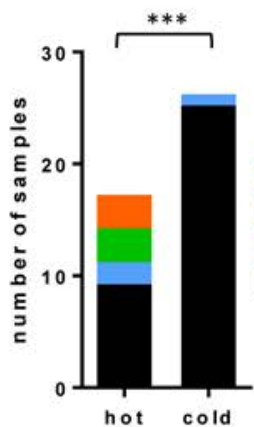

G


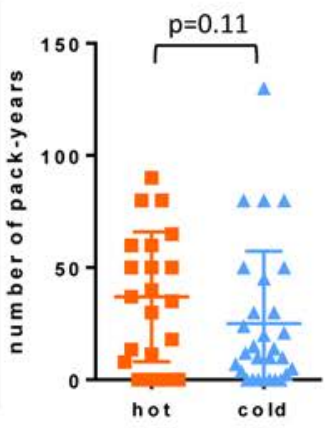

B PD-1+
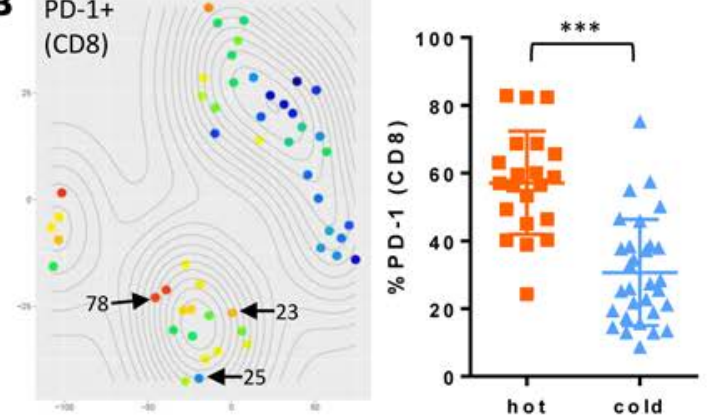

D
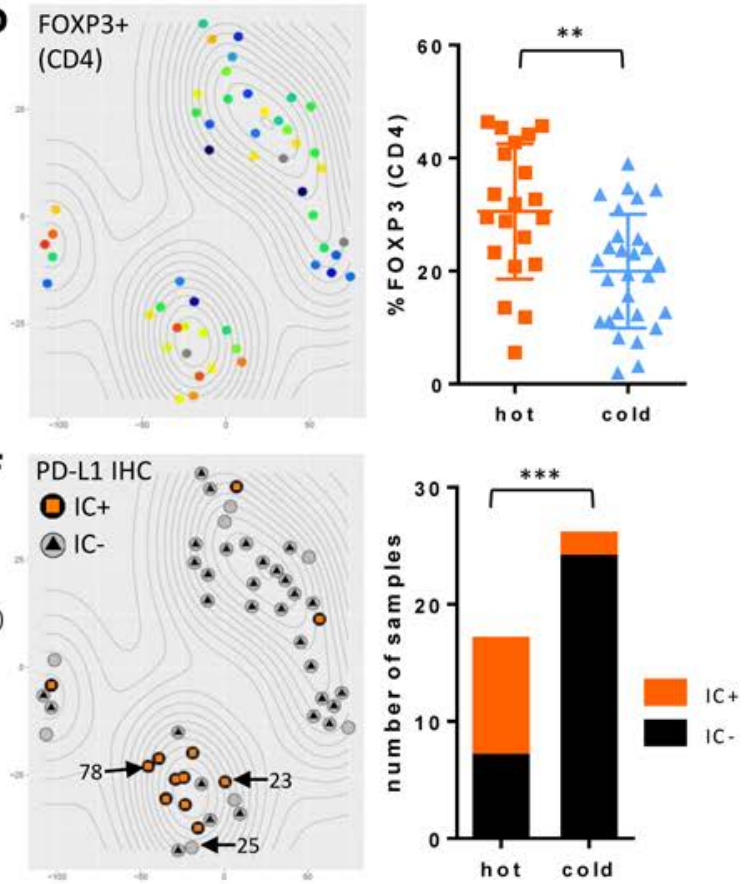

H
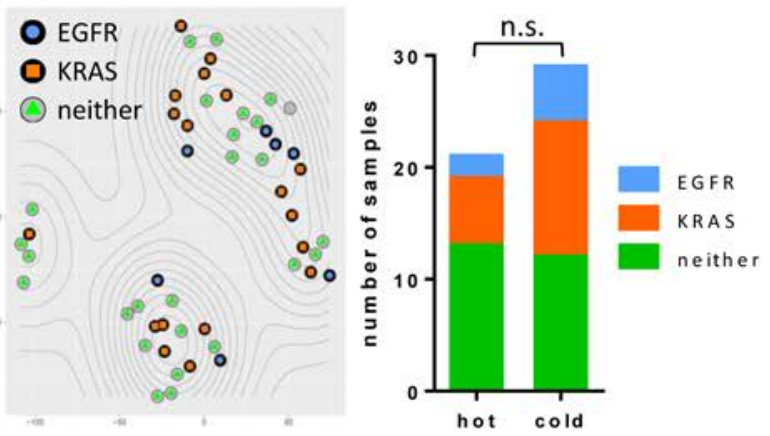

J
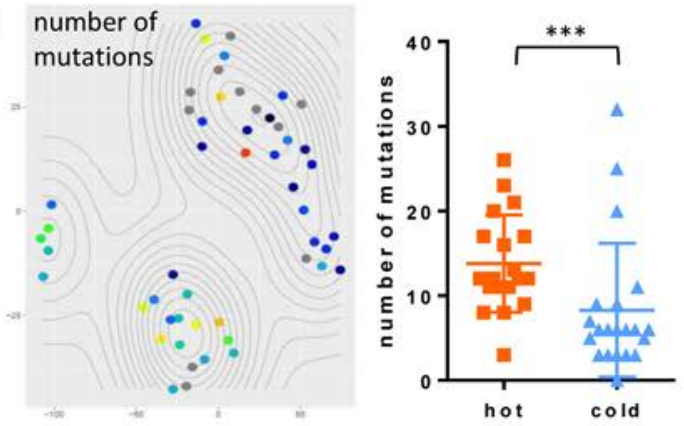
Figure 5. NSCLCs align into immunologically "hot" and "cold" clusters. The t-distributed stochastic neighbor embedding (t-SNE) algorithm assigned NSCLC cases into 2 clusters (dotted ovals). t-SNE plots are identical by NSCLC case coordinate (i.e., each dot is a case and is in the same place in all 10 plots). Percentage of $\mathrm{CD}^{+} \mathrm{T}$ cells of $\mathrm{CD} 3^{+}$Iymphocytes (A), percentage of PD-1 expression on CD8 ${ }^{+} \mathrm{T}$ cells (B), percentage of TIM-3 expression on CD8 $8^{+} \mathrm{T}$ cells $(\mathbf{C})$, and percentage of FOXP3 ${ }^{+}$Tregs of CD4 ${ }^{+}$T cells (D), with gradient color coding of blue (low) to red (high). Percentage of PD-L1 expression on tumor cells by IHC (E), percentage of PD-L1 expression on immune cells by IHC (F), histological subtype (G), oncogene status (H), and smoking status (I) are overlaid on t-SNE plots. (J) Mutation burden is shown with gradient color coding of blue (low) to red (high). Vertical scatter plot statistics are analyzed using unpaired Student's $t$ test and stacked bar graphs are analyzed by Fisher's exact test. ${ }^{* *} P<0.01 ;{ }^{* *} P<0.001$. Mean with SD. Light gray circles on t-SNE plots indicate data not available. Notable examples are indicated by arrows and reference case numbers.

26) and validates our flow clustering methodology (Figure 6). This signature has previously been shown to enrich for tumors responsive to pembrolizumab anti-PD-1 therapy (25). A fully annotated list of significantly upregulated and downregulated genes is available in Supplemental Figure 6.

Given the high concordance among flow cytometry-based t-SNE clustering, PD-L1 IHC, histological classification, smoking history, mutational load, and RNA expression analysis, we sought to find the smallest combination of immune parameters that recapitulated the features of our larger data set as a means to simplify biomarker development. The percentage of $\mathrm{CD}^{+} \mathrm{T}$ cells positive for TIM-3 expression $(P=$ 7.39E-08), the percentage of $\mathrm{CD}^{+} \mathrm{T}$ cells positive for $\mathrm{PD}-1$ expression $(P=2.17 \mathrm{E}-06)$, and the abundance of $\mathrm{CD}^{+} \mathrm{T}$ cells $(P=1.06 \mathrm{E}-04)$ were among the markers most significantly different between "hot" and "cold" clusters. We then recalculated the t-SNE plots based on only those 3 markers and found near-perfect recapitulation of the "hot" and "cold" clusters, with only one case moving from "cold" to "hot"; that one case displayed the highest percentage of PD-1 and TIM-3 expression by CD8 ${ }^{+} \mathrm{T}$ cells within the higher-parameter "cold" cluster (Figure 7A). We conclude that as few as 3 immune parameters, specifically TIM-3 and PD-1 expression on $\mathrm{CD} 8^{+} \mathrm{T}$ cells and presence of $\mathrm{CD} 8^{+} \mathrm{T}$ cells, can be utilized as a proxy to differentiate immunologically "hot" versus "cold" NSCLCs. Furthermore, we propose a model that includes these criteria as a possible metric to assess favorability to immunotherapy (Figure 7B).

NSCLCS are enriched for CD19+ B cells compared with normal lung tissue. We noted high B cell infiltrate in a subset of tumors, which ranged from $0 \%-29.7 \%$ of total live cells (mean $6.159 \% \pm 1.006 \%$, SEM) (Figure 1). We further analyzed tumor-associated B cells by IHC and flow cytometry. The presence of B cell-rich tertiary lymphoid structures (TLSs) adjacent to tumor margins is a good prognostic factor in many cancers, including NSCLC (27). We found abundant TLSs in the tumor sections we surveyed and that the TLS score correlated well with total CD19+ B cell count measured by flow cytometry (Supplemental Figure 7, A-D). We did not, however, see a significant difference in TLSs between "hot" and "cold" clusters (Supplemental Figure 7E). As part of our flow cytometry B cell profiling, we observed a

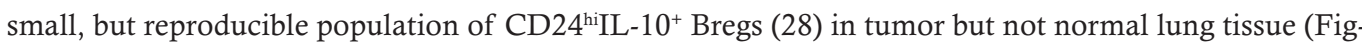
ure $8 \mathrm{~A}$ ). IL-10 is the only definitive marker for Bregs, but it is difficult to measure by flow cytometry due to its low intracellular accumulation. To identify additional phenotypic markers for flow cytometry that could be more sensitive than IL-10 and used as a surrogate for this population, we sorted B cells from a NSCLC tumor with high B cell infiltrate and also B cells from matched normal lung and generated RNA sequencing (RNAseq) data with single-cell resolution $(29,30)$. In total, 130 cells (47 from normal lung, 83 from tumor) passed our quality control filters. Of these 130 cells, we discovered a total of 9 IL-10-expressing B cells, all of which were sorted from the tumor and were not present in normal lung tissue. The top 20 most significantly downregulated and upregulated genes are highlighted by $P$ value as a function of expression (Figure 8B). We did not uncover any novel candidate surface markers that could be used for future phenotypic identification of these IL-10-producing B cells, nor did we observe an enrichment for canonical Breg surface markers CD24, CD27, or CD38. We did, however, perform geneset enrichment analysis (GSEA) using a rank list of all genes that were significantly downregulated or upregulated between IL-10-producing and IL-10- B cells and, by this methodology, observed that these IL-10-producing B cells displayed a transcriptional profile resembling a plasma cell gene signature and not a naive or memory B cell signature (Figure 8C). IL-10-producing B cells also displayed an activated MYC signature. We observed a similar ratio of $\operatorname{Ig} \kappa / \operatorname{Ig} \lambda$ between normal lung B cells and tumor B cells and between IL-10+ B cells and IL-10- B cells, suggesting the absence of clonality in any of these subsets (Supplemental Figure 8). This single-cell transcriptomic analysis of B cells provides initial insight into the biology of NSCLC-associated IL-10-producing B cells.

\section{Discussion}



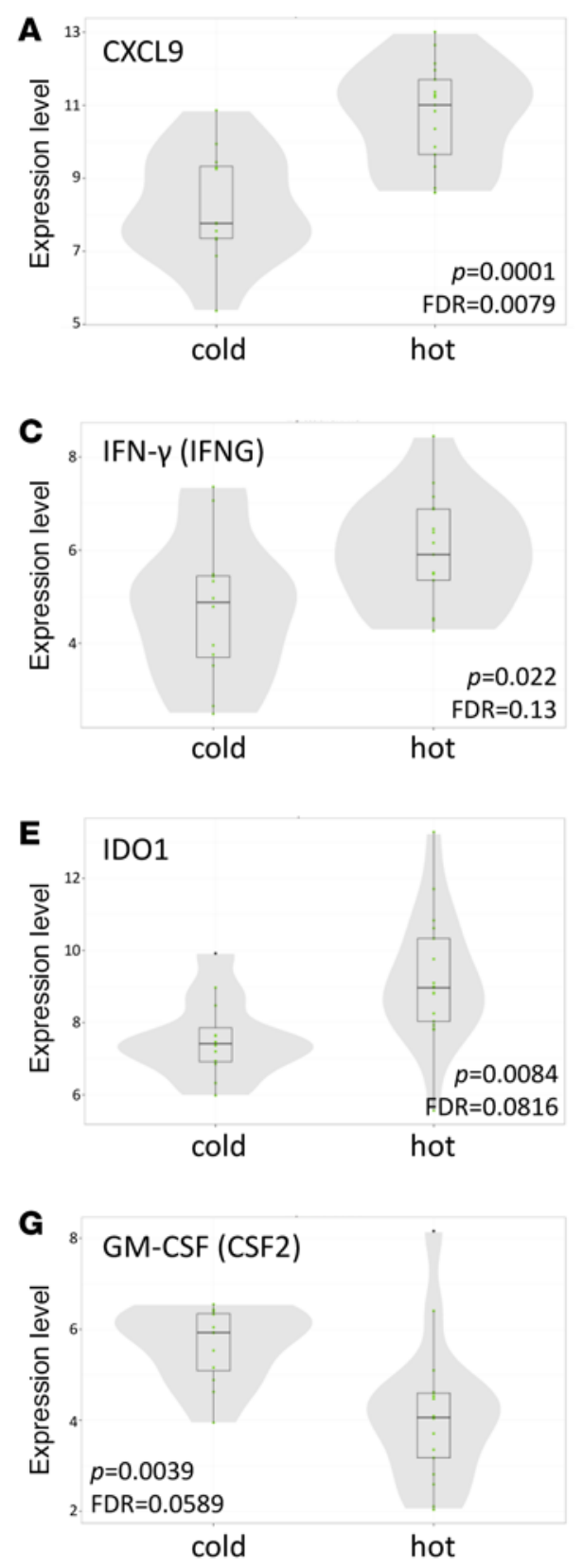
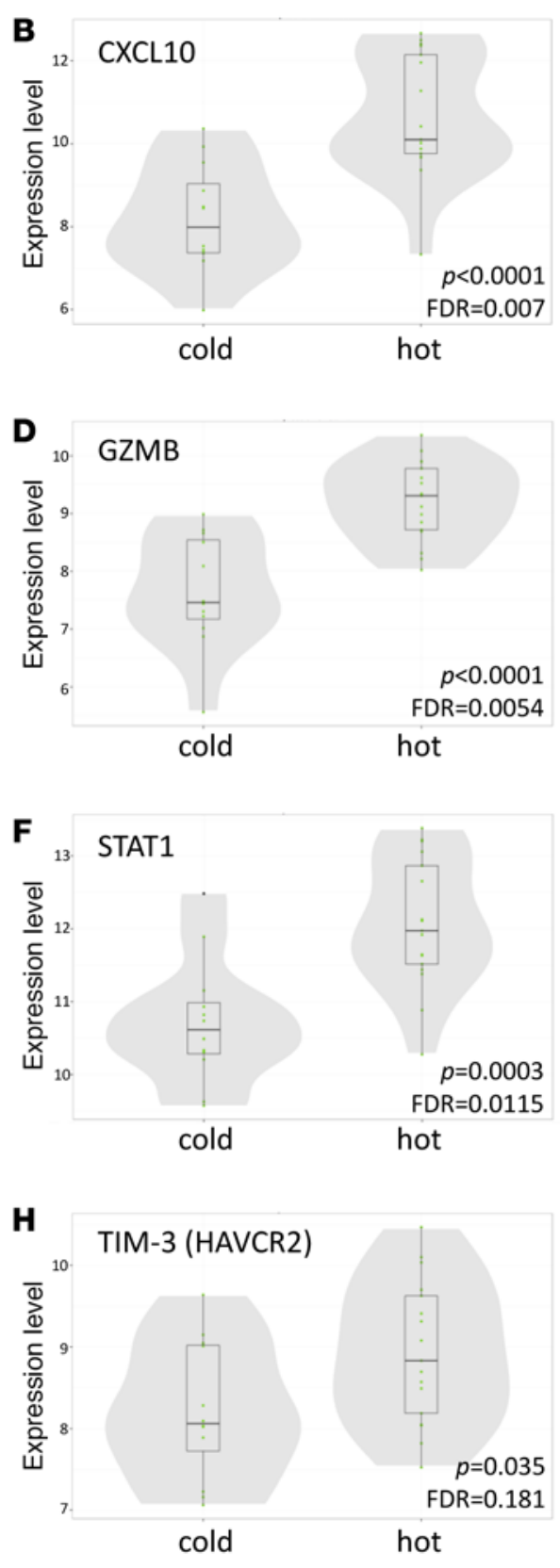

Figure 6. "Hot" cluster is enriched for CTL/Th1-associated genes. Normalized mRNA expression of signature genes is presented for "hot" and "cold" clusters. CXCL9 (A), CXCL10 (B), IFN- $\gamma$ (C), granzyme B (D), IDO1 (E), STAT1 (F), and TIM-3 $(\mathbf{H})$ are upregulated and GM-CSF (C) is downregulated in the "hot" cluster relative to the "cold" cluster. In violin plots, horizontal lines depict medians, with narrow shaded boxes representing the first-to-third interquartile range and vertical lines representing the lower-to-upper adjacent value range. $P$ values were calculated with unpaired Student's $t$ test.

NSCLC is a heterogeneous disease on a molecular level, and, according to our results, it is also heterogeneous immunologically. The presence of leukocytes, specifically $\mathrm{T}$ cells, and their expression of inhibitory receptors targeted by checkpoint blockade likely affects the success of such therapeutics. NSCLCs have higher $\mathrm{CD} 8^{+} \mathrm{T}$ cell infiltrate, particularly $\mathrm{CD} 45 \mathrm{RO}^{+}$ memory $\mathrm{CD}^{+} \mathrm{T}$ cells, relative to normal lung tissue, with no difference between histological subtypes (Figure 3B). NSCLCs also display significantly increased expression of inhibitory receptors $\mathrm{PD}-1$, TIM-3, and CTLA- 4 in both $\mathrm{CD}^{+}$and $\mathrm{CD} 8^{+} \mathrm{T}$ cells relative to normal lung, with little (CTLA-4) or no difference (PD-1, TIM-3) between adenocarcinoma and squamous for $\mathrm{CD}^{+} \mathrm{T}$ cells (Figure 4B). Yet across tumors there are gradients of $\mathrm{T}$ cell infiltrate and expression of inhibitory receptors.

In addition to analysis of individual immunological characteristics on the basis of clinical features, we also analyzed our flow cytometric immunophenotypic data collectively using an unbiased clustering algorithm. The multidimensional reduction methodology t-SNE revealed distinct "hot" versus "cold" clusters (Figure 5). The "hot" cluster was distinguished by the squamous subtype and high $\mathrm{CD} 8^{+} \mathrm{T}$ cell infiltrate, with high coexpression of PD-1 and TIM-3 (Supplemental Figure 2). Tumors in this cluster also displayed high PD-L1 on tumor cells and immune cells by both flow cytometry and PD-L1 IHC, which were highly correlated (Figure 5, E and F, and Supplemental Figure 3). It is interesting that cases with high granulocytic infiltrate and, by extension, lower CD8 ${ }^{+} \mathrm{T}$ cells counts, formed a subgroup within the "hot" cluster, raising the possibility of granulocyte-associated $\mathrm{T}$ cell suppression as a mechanism of immune evasion in this subset. Our data suggest that the total number of $\mathrm{CD}^{+} \mathrm{T}$ cells within the tumor is not sufficiently predictive of the immunophenotype and that it is important to also know if the $\mathrm{T}$ cells express PD-1, TIM-3, both, or other immune checkpoints. CD $8^{+} \mathrm{T}$ cells from granulocyte-high tumors displayed high PD-1 and TIM-3 expression (Figure 5, B and C), indicating a suppressed antitumor immune response (Figure 7B). 
A

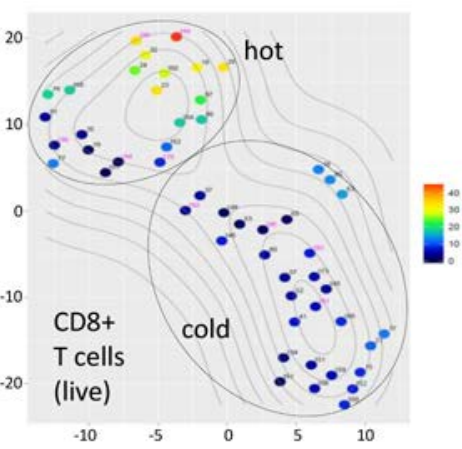

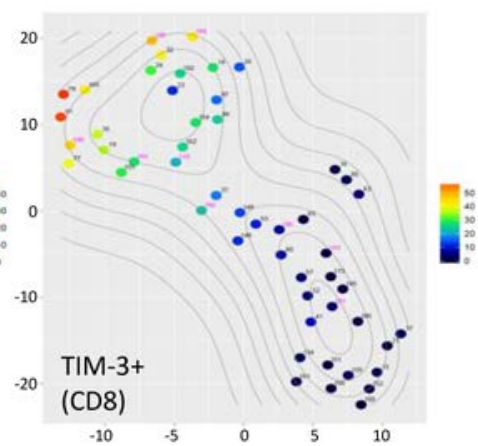

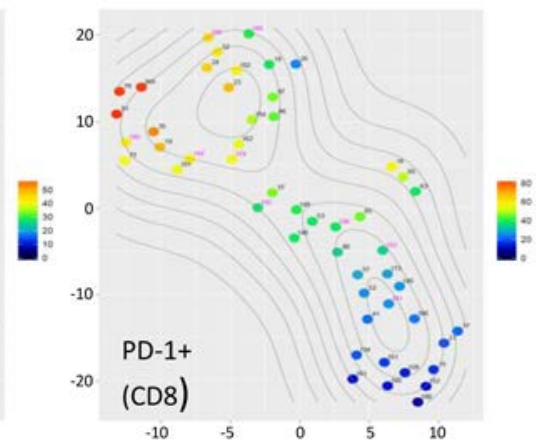

B

Proposed Model

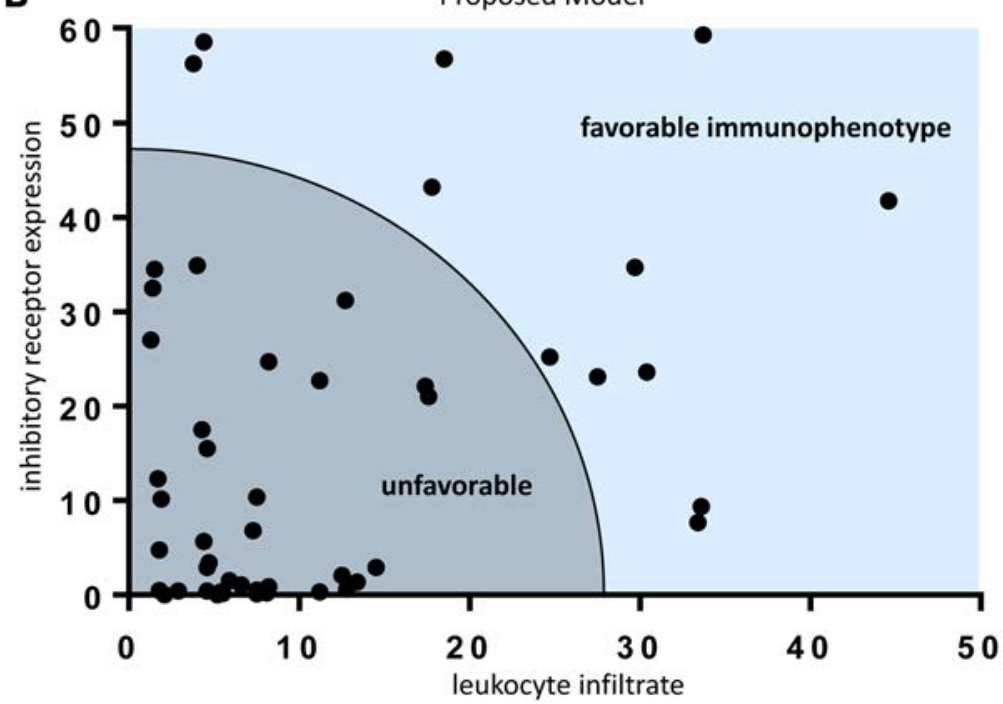

Figure 7. Limited CD8+ $\mathbf{T}$ cell markers predict NSCLC immunophenotypes. (A) NSCLC cases were reanalyzed using t-distributed stochastic neighbor embedding (t-SNE) algorithm based on only 3 parameters: percentage of CD8 ${ }^{+} T$ cells of CD3+ lymphocytes, percentage of TIM-3 expression on CD8 ${ }^{+} T$ cells, and percentage of PD-1 expression on CD8 ${ }^{+} T$ cells. The structure of "hot" and "cold" clusters matched what was observed for multiparameter clustering. (B) Proposed model of immunotherapy-favorable immunophenotype of NSCLC based on limited T cell-intrinsic factors.

In order to corroborate our flow-based clustering and IHC data, we also performed mRNA expression analysis on 29 of our 51 samples. Tumors in the "hot" cluster were enriched for T cell chemoattractants CXCL9 and CXCL10 and CTL/Th1-associated genes IFN- $\gamma$ and granzyme B (Figure 6). Based on our immunophenotypic data, the immunologically "hot" subset of NSCLC is characterized by inflammatory $\mathrm{CD}^{+} \mathrm{T}$ cell infiltrate, PD-L1 IHC positivity, and PD-1 and TIM-3 positivity on CD8 ${ }^{+} \mathrm{T}$ cells and correlated with increased tobacco use, high mutation burden, and squamous histology but not with KRAS or EGFR mutation status (Figure 5 and Figure 6). This collective data supports the concept that there is an ongoing antitumor immune response in the "hot" subset of NSCLC that is suppressed.

While a critical accumulation of tumor-infiltrating leukocytes is likely required for targeted immunotherapies to be successful, simple quantification of leukocyte populations does not capture immune cell phenotypic variation, such as expression of inhibitory receptors and their ligands within the tumor microenvironment. Many immunotherapy biomarker studies perform PD-L1 IHC scoring for tumor cells only and do not include PD-L1 scoring for immune cells. Our data show that $\mathrm{TC}^{-} \mathrm{IC}^{+}$and $\mathrm{TC}^{+}$have similar leukocyte infiltrates (Figure 3D) and similar expression of PD-1 and other immune checkpoints (Figure 4D). Therefore, patients who score as PD-L1- by many IHC tests may be, immunologically speaking, indistinguishable from PD-L1 ${ }^{+}$patients. Relying solely on tumor cell PD-L1 IHC scoring to predict sensitivity to PD-1/PD-L1 blockade, therefore, excludes some patients who may respond to anti-PD-1 therapy. It is these patients with tumor cell and/or immune cell PD-L1+ tumors that populate our "hot" cluster and have the highest levels of $\mathrm{CD}^{+} \mathrm{T}$ cells with expression of PD-1 and TIM-3 (Figure 5). Therefore, inclusion of immune cell PD-L1 scoring into PD-L1 IHC diagnostics may enable that test to more accurately 
A

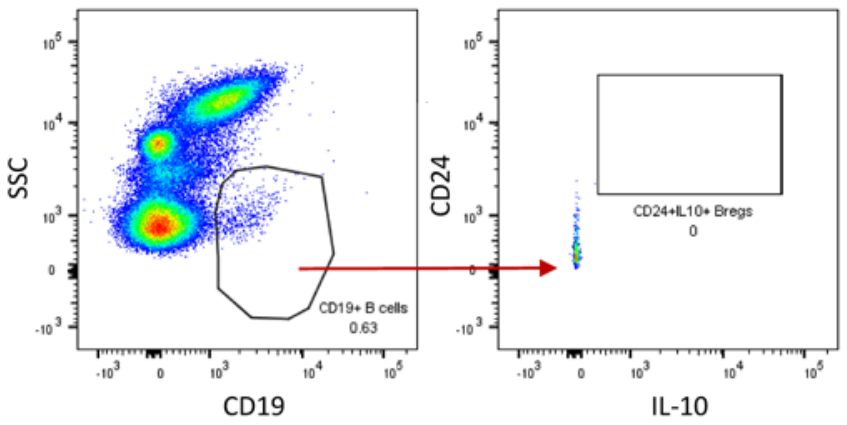

normal lung

B

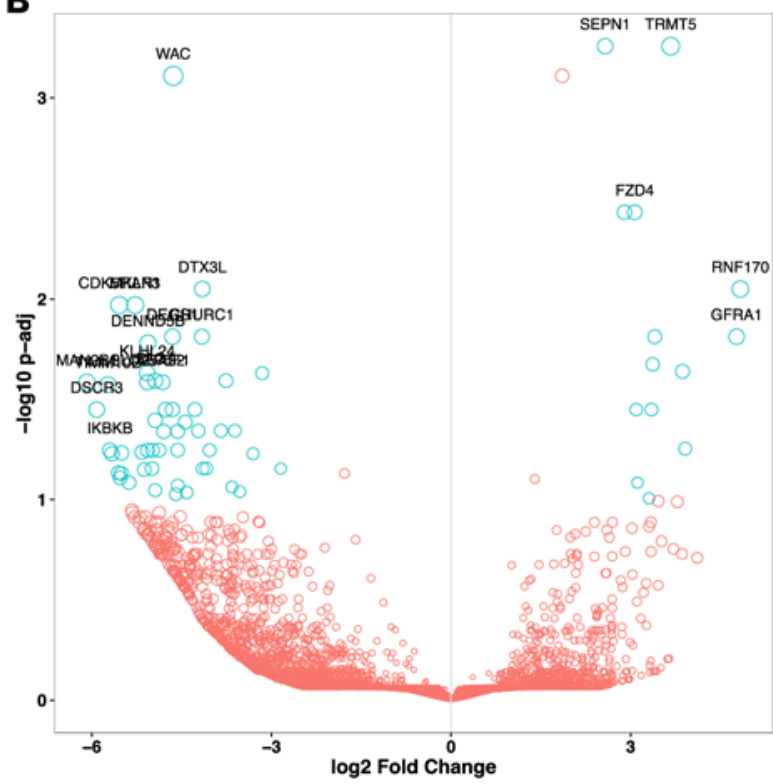

C

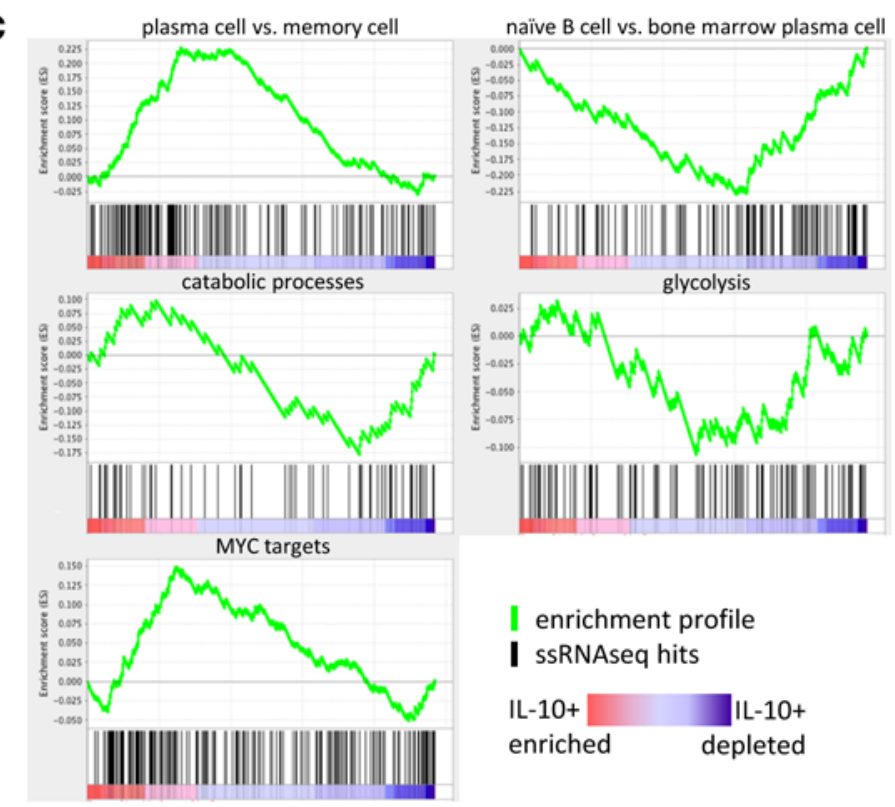

Figure 8. B cell abundance and phenotype in NSCLC. (A) Flow cytometry profiling of NSCLCs revealed a small but reproducible population of IL-10+ B cells in tumor tissue but not normal lung. (B and C) CD19+ B cells were sorted from tumor and normal lung and analyzed by single-cell RNA sequencing. IL-10-producing B cells were present in tumors but not normal lung and display a unique transcriptional profile representative of plasma B cells, oxidative metabolism, and MYC activation.

recapitulate the immunophenotypic features indicative of our "hot" cluster (Figure 5). If the critical factor underlying the response to checkpoint blockade is presence of leukocytes, however, then hybrid IHC/flow cytometry-based readouts may improve upon PD-L1 IHC as an immune biomarker. Only prospective studies with immunoprofiling before and after treatment can answer these questions as to what degree leukocyte abundance and phenotype can predict response to anti-PD-1/PD-L1 therapy.

In our study smoking status and mutational genotyping do not predict immunological features of NSCLC. There are varying reports of associations between mutant EGFR and KRAS and PD-L1 expression, indicating that genomic data may predict response to single-agent anti-PD-1 $(31,32)$. However, we did not observe a correlation between KRAS mutation and immunophenotype or PD-L1 IHC. EGFR tumors were all negative for PD-L1 expression on tumor cells but did not cluster preferentially as immunologically "hot" or "cold" (Figure 5H). Lack of PD-L1 expression by mutant EGFR lung tumors may explain their recently reported low response rates to PD-1 pathway blockade (33). Our data demonstrate that mutation status is not illustrative of immunophenotype and corroborate previous studies in which neither EGFR or KRAS mutations predicted successful response to anti-PD-1 therapy $(34,35)$.

Current and former smokers have higher PD-L1 expression compared with never smokers, and this is thought to explain the higher objective response rate of smokers to anti-PD-L1 therapy (31). Based on previous studies, we expected additional immunological markers to correlate with smoking status. The lack of correlation we found between smoking status and PD-L1 IHC may be due to low sample number 
$\left(9 \mathrm{TC}^{+} \mathrm{PD}-\mathrm{L}^{+}\right)$, but leukocyte abundance and phenotype also did not differ between smokers and never smokers (Figure 3A and Figure 4A). We then broke down smoking status by the more quantitative metric of pack years and found that smoking status weakly correlates with immunophenotype (Figure 5I). It is well established that smokers have higher response rates to anti-PD-1/PD-L1 therapy. The recent study by Rizvi et al. confirmed that mutation burden as well as presence of mutations in DNA repair genes were correlated with anti-PD-1 response (36). One limitation of our study is that we did not perform whole-exome sequencing on our cohort to assess a molecular smoking signature that has been shown to correlate with immunotherapy efficiency better than self-reported smoking status. However, the number of mutations identified using our smaller focused sequencing panel correlated with our immunophenotypic clusters, suggesting the possible use of simple flow-profiling platforms as part of patient stratification for the treatment.

B cells may be protumorigenic or antitumorigenic or even phenotypically heterogeneous within and across tumors (37). In light of the ongoing phase Ib/II clinical trial (NCT02403271) using combination PD-L1 inhibitor (durvalumab) and BTK inhibitor (ibrutinib) for treatment of NSCLC, and the ambiguity as to the role of B cells in NSCLC, we thought a deeper analysis of tumor-associated B cells was warranted. B cells as components of TLSs are good prognostic factors in cancer (27), whereas IL-10-producing Bregs are regarded as negative (28). We found TLSs in 20 of 22 NSCLCs assayed, but there was no association between "hot" or "cold clusters (Supplemental Figure 7E). We consistently identified a small proportion of IL-10-producing B cells in our NSCLC samples that were absent in normal lung tissue (Figure 8A). Bregs are identified by expression of the surface markers $C D 19^{+} \mathrm{CD} 24^{\text {hi }} \mathrm{CD} 38^{\text {hi }} \mathrm{CD} 5^{+} \mathrm{CD} 1 \mathrm{~d}^{\mathrm{hi}}, \mathrm{CD} 24^{\text {hi }} \mathrm{CD} 27^{+}$, and recently Bregs isolated from hepatocellular carcinoma have been shown to be PD $-1{ }^{\text {hi }} C D 5^{\text {hi }} \mathrm{CD} 24^{-/+} \mathrm{C}-$ $\mathrm{D} 27^{\mathrm{hi} /+} \mathrm{CD} 38^{\mathrm{dim}}(28,38)$. We sorted B cells from one tumor and performed RNAseq on single cells. We used the expression of IL-10 as our marker for Bregs, as it is the only consistently expressed gene shared across Breg nomenclatures. IL-10-producing B cells comprised 11\% of tumor-sorted B cells ( 9 of 83 ) and were absent from normal lung-sorted B cells ( 0 of 47). Interestingly, IL-10-producing B cells were not enriched in canonical Breg surface antigens CD24, CD27, or CD38, which may highlight the limitation of profiling of Bregs by flow cytometry and explain the reported diversity of phenotypic markers. This exploratory data set provides a proof of principle for the single-cell expression profiling of tumor-infiltrating B cells and a glimpse at their transcriptional profile. Unlike FOXP3 ${ }^{+}$Tregs, it has been reported that Bregs do not irreversibly lineage commit at an early developmental point but can adopt a regulatory phenotype at various stages along the B cell developmental continuum (28). The IL-10-producing B cells we analyzed by single-cell RNAseq resemble a plasma cell phenotype, with hallmarks of MYC activation and oxidative metabolism (Figure 8, B and C). More analysis is needed to fully deconstruct Breg transcriptional profiles.

It is likely that the presence of leukocytes in the tumor at time of immunotherapy and the expression of inhibitory receptors by tumor-resident T cells — immunologically "hot" versus "cold" — are the critical factors predicting response to anti-PD-1/PD-L1 therapeutics (8). Traditional stratifying clinical criteria, such as oncogenic driver, do not predict immunophenotype and, therefore, may not inform the use of checkpoint blockade. NSCLCs clearly fall into immunologically "hot" or "cold" clusters, and only integration of flow-based, immunohistochemical, and transcriptomic immunophenotyping can differentiate between the two. It is tantalizing to hypothesize that as few as 3 markers (Figure 7) can recapitulate more comprehensive immunophenotyping to determine whether a NSCLC is "hot" or "cold" and, hence, more amenable to immunomodulatory therapy. This study defines the broad spectrum of immunophenotypes that constitute NSCLC and sets the stage for future prospective studies to identify immune cell-based biomarker signatures predictive of response and resistance to immunotherapies.

\section{Methods}

Tumor preparation, flow cytometry, and antibodies. Fresh tissue was minced in a $10-\mathrm{cm}$ dish then resuspended in dissociation buffer consisting of RPMI (Life Technologies) +10\% FBS (HyClone), $100 \mathrm{U} / \mathrm{ml}$ collagenase type IV (Life Technologies), and $50 \mu \mathrm{g} / \mathrm{ml}$ DNase I (Roche) at a ratio of $5 \mathrm{ml}$ of dissociation buffer to $500 \mathrm{mg}$ of sample. Suspension was incubated at $37^{\circ} \mathrm{C}$ for 45 minutes and then further dissociated by being passed through a syringe. Red blood cells were removed from samples using red blood cell lysis buffer (BioLegend). Samples were pelleted and then resuspended in fresh RPMI $+10 \%$ FBS and strained over a $70-\mu \mathrm{m}$ filter. Cells were incubated with the Live/Dead Fixable Yellow Dead Cell Stain Kit (Life Technologies) for 8 minutes in the dark at room temperature or Live/Dead Fixable Zombie NIR (Biolegend) for 5 minutes in the dark at room temperature in FACS buffer (PBS $+2 \%$ FBS) at a ratio of $250 \mu \mathrm{L} / \mathrm{D} 1 \mathrm{X}$ dilution to 100 
mg of original sample weight. Surface marker and intracellular staining were performed according to the manufacturer's protocols (eBioscience). FcR was blocked prior to surface antibody staining using Human FcR Blocking Reagent (Miltenyi). Cells were fixed in 1\% PBS $+2 \%$ FBS and washed prior to analysis on a BD FACSCanto II HTS cell analyzer or BD LSRFortessa with FACSDiva software (BD Biosciences). Data were analyzed using FlowJo software version 10.0.8. Cell viability was determined by negative live/ dead staining. Antibodies were specific for the following human markers: CD3 (HIT3a; UCHT1), CD8 (RPA-T8), CD14 (M5E2; MphiP9), CD24 (ML5), CD45 (HI30), CD56 (B159), CCR7 (150503), EpCAM (EBA-1), HLA-DR (G46-6), PD-1 (EH12.1), and IgG1 isotype control (MOPC-21) from BD Biosciences; CD3 (UCHT1), CD4 (RPA-T4), CD14 (M5E2), CD15 (W6D3), CD16 (3G8), CD19 (HIB19), CD20 (2H7), CD21 (Bu32), CD25 (BC96), CD27 (M-T271), CD33 (WM53), CD38 (HIT2), CD40L (26-33), CD45 (HI30), CD45RA (HI100), CD45RO (UCHL1), CD56 (HCD56; 5.1H11), CD66b (G10F5), CD69 (FN50), CD83 (HB15e), CD123 (6H6), CD160 (BY55), CD163 (GHI/61), CTLA-4 (L3D10), CXCR5 (J252D4), EpCAM (9C4), HMGB1 (3E8), IgM (MHM-88), Ki-67 (Ki-67), PD-1 (EH12.2H7), PD-L1 (29E.2A3), PD-L2 (24F.10C12), TIM-3 (F38-2E2), NKG2D (1D11), NKp46 (9E2), IgG2a isotype control (MOPC-173), IgG2b isotype control (MPC-11), and IgG1 isotype control (MOPC-21) from BioLegend; Pan-cytokeritin (C11) and PD-L1 (E1L3N) from Cell Signaling Technologies; CD45 (2D1), FOXP3 (236A/E7), and IL-10 (236A/E7) from Affymetrix/eBioscience; and LAG3 (polyclonal) and isotype control (polyclonal) from R\&D Systems.

Collection of surgical samples. NSCLC tumors, matched normal lung, and, where available, peripheral blood, were obtained from 51 patients. Tumor samples were collected into sterile medium (DMEM +FBS) and stored on ice before dissociation, which was begun 30-45 minutes after resection. The histological subtype of tumors was confirmed by a board-certified pathologist with expertise in thoracic malignancies (LMS). Patients from all disease stages were selected in an unbiased manner over a 2-year period. The prospective nature of this study assumed heterogeneity in immune profiles.

Tumor genotyping via digital droplet PCR. Cryosections from tumor tissue samples underwent DNA extraction using the Qiagen DNeasy Blood \& Tissue Kit according to the manufacturer's protocol. DNA was then eluted in $102 \mu \mathrm{l}$ of AVE buffer and stored at $-80^{\circ} \mathrm{C}$ until genotyping was performed. Digital droplet PCR-based (ddPCR-based) genotyping was performed for EGFR mutations, specifically exon 19 del and L858R, and KRAS mutations, specifically condon 12 mutation and G13D. The development of this assay has been previously described (39). Briefly, diluted tumor DNA was emulsified into approximately 20,000 droplets and mixed with appropriate primers/probes and PCR mastermix; then PCR was carried out to endpoint. Droplets were then read in a flow cytometer (QX200 Droplet Reader, Bio-Rad), and fluorescence signal was quantified in order to determine the number of copies of mutant and wild-type alleles per $\mu 1$ of the reaction. Extracted DNA was quantified by UV-Vis spectrophotometers and diluted to 2,000 genetic equivalents/ $\mu$ (Thermo Scientific). Genotyping of tumor DNA was then performed using ddPCR reagents (Bio-Rad) and primer/probe mixes, which were custom-made by Life Technologies. For EGFR L858R assay, primer sequences were as follows: forward, 5'-GCAGCATGTCAAGATCACAGATT-3', reverse, 5'-CCTCCTTCTGCATGGTATTCTTTCT-3'; probe sequences were as follows: 5'-VIC-AGTTTGGCCAGCCCAA-MGB-NFQ-3', 5'-FAM-AGTTTGGCCCGCCCAA-MGB-NFQ-3'. For EGFR del19 ddPCR assay, primer sequences were as follows: forward, 5'-GTGAGAAAGTTAAAATTCCCGTC-3', reverse, 5'-CACACAGCAAAGCAGAAAC-3'; probe sequences were as follows: 5'-FAM-AGGAATTAAGAGAAGCAACATC-MGB-3' (ex19 deletion hotspot probe), 5'-VIC-ATCGAGGATTTCCTTGTTG-MGB-3' (ex19 reference probe). For KRAS G12A assay, primer sequences were as follows: forward, 5'-GCCTGCTGAAAATGACTGAATATAAACT-3', reverse, 5'-GCTGTATCGTCAAGGCACTCTT-3'; probe sequences were as follows: 5'-VIC-TTGGAGCTGGTGGCGTA-MGB-NFQ-3', 5'-FAM-TTGGAGCTGCTGGCGTA-MGB-NFQ-3'. For KRAS G12C assay, primer sequences were as follows: forward, 5'-GCCTGCTGAAAATGACTGAATATAAACT-3', reverse, 5'-GCTGTATCGTCAAGGCACTCTT-3'; probe sequences were as follows: 5'-VIC-TTGGAGCTGGTGGCGTA-MGB-NFQ-3', 5'-FAM-TTGGAGCTTGTGGCGTA-MGB-NFQ-3'. For KRAS G13D assay, primer sequences were as follows: forward, 5'-GCCTGCTGAAAATGACTGAATATAAACT-3', reverse, 5'-GAATTAGCTGTATCGTCAAGGCACT-3'; probe sequences were as follows: 5'-VIC-CTTGCCTACGCCACCAG-MGB-NFQ-3', 5'-FAM-CTTGCCTACGTCACCAG-MGB-NFQ-3'. For KRAS G12D assay, primer sequences were as follows: forward, 5'-GCCTGCTGAAAATGACTGAATATAAACT-3', reverse, 5'-GCTGTATCGTCAAGGCACTCTT-3'; probe sequences were as follows: 5'-VIC-TTGGAGCTGGT- 
GGCGTA-MGB-NFQ-3', 5'-FAM-TTGGAGCTGATGGCGTA-MGB-NFQ-3'. For KRAS G12V assay, primer sequences were as follows: forward, 5'-GCCTGCTGAAAATGACTGAATATAAACT-3', reverse, 5'-GCTGTATCGTCAAGGCACTCTT-3'; probe sequences were as follows: 5'-VIC-TTGGAGCTGGTGGCGTA-MGB-NFQ-3', 5'-FAM-TTGGAGCTGTTGGCGTA-MGB-NFQ-3'. For KRAS G12S assay, primer sequences were as follows: forward, 5'-GCCTGCTGAAAATGACTGAATATAAACT-3', reverse, 5'-GCTGTATCGTCAAGGCACTCTT-3'; probe sequences were as follows: 5'-VIC- TTGGAGCTGGTGGCGTA-MGB-NFQ-3'， 5'-FAM-TAGTTGGAGCTAGTGGCGTA-MGB-NFQ-3'. Analysis of the ddPCR data was performed with QuantaSoft analysis software (Bio-Rad) that accompanied the droplet reader. Positive and negative clusters were set using the FAM and VIC thresholds based on the amplitude of positive controls that were ran concomitantly with each assay.

$m R N A$ analysis. Total RNA was extracted using the Qiagen RNeasy kit or Arcturus PicoPure RNA Isolation Kit. RNA quality and concentration was assessed using an Agilent Bioanalyzer 2100. For each sample, mRNA transcript abundance for 770 genes of interest was quantified using the Nanostring nCounter Human PanCancer Immune Profiling Panel according to the manufacturer's protocol from $100 \mathrm{ng}$ of total RNA and analyzed using nSolver 2 software and the HumanPanCancerImmunology_1.0.36 analysis module. Differential expression of genes in response to "hot" versus "cold" sample breakdown was performed in the Advanced Analysis module of nSolver. For each gene, a single linear regression was fit using all selected covariates to predict expression. Output is shown with nonadjusted $P$ value as well as Benjamini-Hochberg FDR. Univariate box/violin plots were generated via Gene Descriptive Analyses module. Nanostring array data have been deposited in the NCBI GEO public data repository (reference series accession GSE84799).

$I H C$. Four-micron-thick sections of formalin-fixed, paraffin-embedded tissue were baked at $37^{\circ} \mathrm{C}$ overnight, deparaffinized, and rehydrated. Peroxidase activity was blocked with $1.5 \%$ hydrogen peroxide in methanol for 10 minutes. Antigen retrieval was performed in a Decloaking Chamber NxGen pressure cooker (Biocare Medical) at $120^{\circ} \mathrm{C}$ in Dako Target Retrieval Solution (Dako). For PD-L1/Pu.1 double staining, sections were first incubated with anti-PU.1 (BD Bioscience) at 1:100 for 40 minutes at room temperature. Anti-mouse Dako EnVision+ System-HRP (DAB) was used for the detection. Following the wash, sections were incubated with anti-PD-L1 (CST) at 1:200 for 40 minutes at room temperature. PowerVision Poly-AP Anti-Rabbit IgG (Leica)with Permanent Red was used for the detection. For B cell-specific activator protein (BSAP or PAX5)/CD3 double IHC staining, sections were first incubated with anti-PAX5 (Abcam) at 1:30 for 40 minutes at room temperature. Dako EnVision+ anti-Rabbit (DAB) was used for the detection. Sequentially sections were incubated with anti-CD3 (Dako) at 1:300 for 40 minutes at room temperature. Leica's PowerVision Poly-AP Anti-Rabbit IgG with Permanent Red was used for the detection. All sections were counterstained with Mayer's Hematoxylin.

PD-L1 expression in tumor cells was considered positive if $\geq 1 \%$ of tumor cells had membranous staining of any intensity. Pu. 1 is a transcription factor of B lymphocytes and cell myeloid lineages, among which it is overexpressed in monocytes, histiocytes, and dendritic cells. Pu.1 expression is restricted to the nucleus and was examined simultaneously to better assess PD-L1 staining in immune cells. Alveolar macrophages have been shown to express multiple markers nonspecifically and were excluded from analysis.

BSAP is a transcription factor expressed in the nuclei of pro, pre, and mature B cells. B cell quantification was performed using the Positive Pixel Count v9 algorithm by Aperio (Leica). Intensity of weak pixels (Iwp[High] parameter) was changed from 220 to 180 to adjust to hematoxylin staining on nuclei as a proxy for total cell count. DAB staining for BSAP was counted as the number of strong positive pixels. Permanent red staining for CD3 was detected as positive pixels; however, some areas of darker hematoxylin as well as nonspecific staining in alveolar macrophages were also detected as positive pixels, preventing correct quantification of CD3 staining. The BSAP score was generated by dividing the number of strong positive pixels by the area score (number of negative + positive + strong positive pixels multiplied by 10e8).

TLSs are lymph node-like arrangements of several immune cell types (primarily B and T lymphocytes) recently shown to play an important role in tumor microenvironment (40). TLS were counted manually on Aperio-scanned slides with the minimal zoom of $\times 0.5$. At this resolution, 130 - to $200-\mu \mathrm{m}$ clusters of $\mathrm{B}$ and T cells were visible and counted as small, 200 300 $\mu \mathrm{m}$ clusters of B and T cells were counted as medium, and $>300 \mu \mathrm{m}$ clusters of B and T cells were counted as large TLSs (Supplemental Figure 7). TLS score is reported as the number of small TLSs plus the number of medium TLSs multiplied by 2, plus the number of large TLSs multiplied by 3 , and divided by area score from B cell quantification. All the slides were eval- 
uated and scored blinded to clinical data.

Single-cell RNAseq. Live CD $45^{+} \mathrm{CD} 19^{+}$cells were sorted directly into $2 \mu \mathrm{l}$ of Qiagen TCL buffer in Eppendorf TWIN.TEC skirted 96-well plates. The SmartSeq2 libraries were prepared according to the SmartSeq2 protocol $(29,41)$ with some modifications (42). Briefly, total RNA was purified using RNASPRI beads. Poly $(A)+$ mRNA was converted to cDNA, which was then amplified. cDNA was subjected to transposon-based fragmentation that used dual indexing to barcode each fragment of each converted transcript with a combination of barcodes specific to each sample. In the case of single-cell sequencing, each cell was given its own combination of barcodes. Barcoded cDNA fragments were then pooled prior to sequencing. Paired-end (PE) sequencing was carried out twice with 25-bp reads, with an additional 8 cycles for each index. The Smart-Seq2 data was processed at the Broad Technology Labs according to established computational pipeline. Data were separated by barcode and aligned using Tophat version 2.0.10 (43) with default settings. PE 25-bp reads were mapped to the UCSC human genome (hg19) by Bowtie2/Tophat (44) using the Broad pipeline. Only cells that had a minimum of $100,000 \mathrm{PE}$ reads, with at least $20 \%$ aligning to the genome, were retained for further analysis. FeatureCounts (45) was used to count features based on the Gencode v19 (http://www.gencodegenes.org/) transcriptome annotation. Features that were not detected in more than 10 cells were removed. High-quality single cells were further selected based on feature complexity, read distribution, and number of genes detected. In total 47 normal and 83 tumor cells were retained in this process. DESeq2 (46) was used to detect differentially expressed genes between groups based on raw counts. Counts were normalized according to their library size and displayed as $\log _{2}$ (normalized counts +1 ). IL-10 high and low clusters were classified based on Kmeans clustering on $\log _{2}$ (normalized counts) excluding cells with no detectable IL-10 expression. To identify positive or negative enriched biological or molecular signatures between the IL-10 high and low population we applied the GSEA tool (47) on the DESeq2-generated list of differentially expressed genes. This list was preranked based on the multiplication value of $\log _{2}$ fold change and $-\log _{10}$ (adjusted $P$ value). To estimate clonality of B cell Ig-producing cells, we summed all reads of genes that are located in $\kappa$ and $\lambda$ loci, respectively, as well as all reads of IGK and IGL genes annotated by Gencode v19, and calculated the $\kappa / \lambda$ ratio (Igא/IGg $\lambda$ ). Cells with a ratio between 1:3 and 3:1 were considered to express both loci. Cells with ratios $>3: 1$ or $<1: 3$ were defined $\kappa$ - or $\lambda$-producing cells, respectively. Single-cell RNAseq NGS data have been deposited in the NCBI GEO public data repository (reference series accession GSE84799).

Statistics. The unsupervised nonlinear dimension reduction method t-SNE $(19,20)$ was applied to investigate in reduced dimension space how 51 tumors are located in relation to each other based on multiparametric flow cytometry data. t-SNE denotes a tree-based algorithm, which minimizes the divergence of neighborhood closeness moving from high dimensions to low dimensions. For the embedding, 15 parameters were used, which are listed in Supplemental Figure 9. $P$ values of less than 0.05 were considered significant. Unpaired, 2-tailed Student's $t$ test was used to assess significance. More in-depth descriptions are given in individual figure legends.

Study approval. The present studies were reviewed and approved by the Dana-Farber/Harvard Cancer Center (DF/HCC) institutional review board (Boston, Massachusetts, USA) under protocol 98-063 and all were performed in accordance with relevant guidelines and regulations. Written informed consent was obtained from all subjects prior to participation in this study. Informed consent by patients to DF/HCC protocol 02-180 enabled collection of clinical and demographic data, genomic characterization by OncoPanel, and analysis of tissue by IHC.

\section{Author contributions}

PHL and EVI wrote the manuscript. PHL, EVI, MMA, REJ, LK, CA, NBF, and MMK generated data. $\mathrm{HL}$ and RD provided advanced statistical analysis. MMA and AS provided NGS OncoPanel data. WGR and RB acquired patient samples. PHL, EVI, MMA, GSHS, CPP, AKR, GCY, DWK, PAJ, PSH, FSH, $\mathrm{JME}, \mathrm{MAB}, \mathrm{AJB}$, and KKW designed the experiments. LMS analyzed histology.

\section{Acknowledgments}

We thanks the patients and their families for granting us access to these research samples. We thank the Broad Technology Labs for preparing Smart-Seq2 libraries and sequencing. We thank the Dana-Farber Cancer Institute Hematologic Neoplasia Flow Cytometry core facility and, in particular, Suzan Lazo, Alexander Heubeck, and Hanna Sobon for technical support. We also thank Zach Herbert and Leslie 
Grimmett from the Molecular Biology Core Facility Genomics group for Nanostring analysis. We thank Mei Zhang from the Pathology Department at Brigham and Women's Hospital for immunohistochemical staining. This work was supported by The Robert A. and Renée E. Belfer Family Foundation, the Expect Miracles Foundation, the Starr Cancer Consortium, Stand Up To Cancer - American Cancer Society Lung Cancer Dream Team Translational Research Grant (Grant Number: SU2C-AACR-DT17-15), the Conquer Cancer Foundation, the International Association for the Study of Lung Cancer, the National Cancer Institute (R01 CA205150), and the Damon Runyon Cancer Research Foundation.

Address correspondence to: Kwok-Kin Wong, Dana-Farber Cancer Institute, 450 Brookline Avenue, Boston, Massachusetts 02215, USA. Phone: 617.632.6084; E-mail: kwok-kin_wong@dfci.harvard.edu.

1. Borghaei $\mathrm{H}$, et al. Nivolumab versus docetaxel in advanced nonsquamous non-small-cell lung cancer. $N$ Engl J Med. 2015;373(17):1627-1639.

2. Topalian SL, et al. Safety, activity, and immune correlates of anti-PD-1 antibody in cancer. NEngl J Med. 2012;366(26):2443-2454.

3. Garon EB, et al. Pembrolizumab for the treatment of non-small-cell lung cancer. N Engl J Med. 2015;372(21):2018-2028.

4. EER Stat Fact Sheets: Lung and Bronchus Cancer. National Cancer Institute. http://seer.cancer.gov/statfacts/html/lungb.html Accessed August 30, 2016

5. Rizvi NA, et al. Activity and safety of nivolumab, an anti-PD-1 immune checkpoint inhibitor, for patients with advanced, refractory squamous non-small-cell lung cancer (CheckMate 063): a phase 2, single-arm trial. Lancet Oncol. 2015;16(3):257-265.

6. Brahmer J, et al. Nivolumab versus docetaxel in advanced squamous-cell non-small-cell lung cancer. $N$ Engl J Med. 2015;373(2):123-135.

7. Gettinger SN, et al. Overall Survival and Long-Term Safety of Nivolumab (Anti-Programmed Death 1 Antibody, BMS-936558, ONO-4538) in Patients With Previously Treated Advanced Non-Small-Cell Lung Cancer. J Clin Oncol. 2015;33(18):2004-2012.

8. Herbst RS, et al. Predictive correlates of response to the anti-PD-L1 antibody MPDL3280A in cancer patients. Nature. 2014;515(7528):563-567.

9. Taube JM, et al. Association of PD-1, PD-1 ligands, and other features of the tumor immune microenvironment with response to anti-PD-1 therapy. Clin Cancer Res. 2014;20(19):5064-5074.

10. Yu H, Boyle TA, Zhou C, Rimm DL, Hirsch FR. PD-L1 Expression in Lung Cancer. J Thorac Oncol. 2016;11(7):964-975.

11. Koyama S, et al. Adaptive resistance to therapeutic PD-1 blockade is associated with upregulation of alternative immune checkpoints. Nat Commun. 2016;7:10501.

12. Black CC, Turk MJ, Dragnev K, Rigas JR. Adenocarcinoma contains more immune tolerance regulatory t-cell lymphocytes (versus squamous carcinoma) in non-small-cell lung cancer. Lung. 2013;191(3):265-270.

13. Liu J, et al. Aberrant frequency of IL-10-producing B cells and its association with Treg and MDSC cells in non small cell lung carcinoma patients. Hum Immunol. 2016;77(1):84-89.

14. Ji M, et al. PD-1/PD-L1 expression in non-small-cell lung cancer and its correlation with EGFR/KRAS mutations. Cancer Biol Ther. 2016;17(4):407-413.

15. Dela Cruz CS, Tanoue LT, Matthay RA. Lung cancer: epidemiology, etiology, and prevention. Clin Chest Med. 2011;32(4):605-644

16. McGranahan N, et al. Clonal neoantigens elicit T cell immunoreactivity and sensitivity to immune checkpoint blockade. Science. 2016;351(6280):1463-1469.

17. Galon J, Angell HK, Bedognetti D, Marincola FM. The continuum of cancer immunosurveillance: prognostic, predictive, and mechanistic signatures. Immunity. 2013;39(1):11-26.

18. Cesano A. nCounter(®) PanCancer Immune Profiling Panel (NanoString Technologies, Inc., Seattle, WA). J Immunother Cancer. 2015;3:42.

19. Murtagh F. Multidimensional Clustering Algorithms. Fionn Murtagh. http://www.multiresolutions.com/strule/old-articles/ MultidimensionalClusteringAlgorithms_FMurtagh.pdf. Accessed: August 9, 2016.

20. Murtagh F, Legendre P. Ward's hierarchical agglomerative clustering method: which algorithms implement Ward's criterion? $J$ Classif. 2014;31(3):274-295.

21. Cryan JB, et al. Clinical multiplexed exome sequencing distinguishes adult oligodendroglial neoplasms from astrocytic and mixed lineage gliomas. Oncotarget. 2014;5(18):8083-8092.

22. Wagle N, et al. High-throughput detection of actionable genomic alterations in clinical tumor samples by targeted, massively parallel sequencing. Cancer Discov. 2012;2(1):82-93.

23. Van Der Maaten L. Accelerating t-SNE Using Tree-based Algorithms. J Mach Learn Res. 2014;15(1):3221-3245.

24. Frampton GM, et al. Assessment of tumor mutation burden from $>60,000$ clinical cancer patients using comprehensive genomic profiling. ASCO University. http://meetinglibrary.asco.org/content/167202-176. Accessed August 9, 2016.

25. Sinkus AG, Kuliev AM. [Chromosomal damage in a series of cell generations following application of rubomycin $C$ to cultured human cells]. Biull Eksp Biol Med. 1972;73(11):112-114.

26. Ananiev T, Vasilev Z, Nalbaiski B. [Congenital abnormalities of the uterus]. Akush Ginekol (Sofiia). 1974;13(2):136-143.

27. Germain C, Gnjatic S, Dieu-Nosjean MC. Tertiary lymphoid structure-associated B cells are key players in anti-tumor immunity. Front Immunol. 2015;6:67.

28. Balkwill F, Montfort A, Capasso M. B regulatory cells in cancer. Trends Immunol. 2013;34(4):169-173.

29. Picelli S, Faridani OR, Björklund AK, Winberg G, Sagasser S, Sandberg R. Full-length RNA-seq from single cells using Smartseq2. Nat Protoc. 2014;9(1):171-181.

30. Shalek AK, et al. Single-cell RNA-seq reveals dynamic paracrine control of cellular variation. Nature. 2014;510(7505):363-369. 
31. Calles A, et al. Expression of PD-1 and its ligands, PD-L1 and PD-L2, in smokers and never smokers with KRAS-mutant lung cancer. J Thorac Oncol. 2015;10(12):1726-1735.

32. Lastwika KJ, et al. Control of PD-L1 expression by oncogenic activation of the AKT-mTOR pathway in non-small cell lung cancer. Cancer Res. 2016;76(2):227-238.

33. Gainor JF et al. EGFR mutations and alk rearrangements are associated with low response rates to PD-1 pathway blockade in non-small cell lung cancer (NSCLC): A Retrospective Analysis [published online ahead of print May 25, 2016]. Clin Cancer Res. doi:10.1158/1078-0432.CCR-15-3101.

34. Creelan BC. Update on immune checkpoint inhibitors in lung cancer. Cancer Control. 2014;21(1):80-89.

35. Garon EB et al. Abstract A20: MK-3475 monotherapy for previously treated non-small cell lung cancer (NSCLC): Preliminary safety and clinical activity. Clin Cancer Res. 2014;20(2 Supplement):A20-A20.

36. Rizvi NA, et al. Cancer immunology. Mutational landscape determines sensitivity to PD-1 blockade in non-small cell lung cancer. Science. 2015;348(6230):124-128.

37. Fremd C, Schuetz F, Sohn C, Beckhove P, Domschke C. B cell-regulated immune responses in tumor models and cancer patients. Oncoimmunology. 2013;2(7):e25443.

38. Xiao X, et al. PD-1hi identifies a novel regulatory B-cell population in human hepatoma that promotes disease progression. Cancer Discov. 2016;6(5):546-559.

39. Oxnard GR, et al. Noninvasive detection of response and resistance in EGFR-mutant lung cancer using quantitative next-generation genotyping of cell-free plasma DNA. Clin Cancer Res. 2014;20(6):1698-1705.

40. Goc J, Fridman WH, Sautès-Fridman C, Dieu-Nosjean MC. Characteristics of tertiary lymphoid structures in primary cancers. Oncoimmunology. 2013;2(12):e26836.

41. Picelli S, Björklund ÅK, Faridani OR, Sagasser S, Winberg G, Sandberg R. Smart-seq2 for sensitive full-length transcriptome profiling in single cells. Nat Methods. 2013;10(11):1096-1098.

42. Trombetta JJ, Gennert D, Lu D, Satija R, Shalek AK, Regev A. Preparation of single-cell RNA-Seq libraries for next generation sequencing. Curr Protoc Mol Biol. 2014;107:4.22.1-4.2217.

43. Kim D, Pertea G, Trapnell C, Pimentel H, Kelley R, Salzberg SL. TopHat2: accurate alignment of transcriptomes in the presence of insertions, deletions and gene fusions. Genome Biol. 2013;14(4):R36.

44. Trapnell C, Pachter L, Salzberg SL. TopHat: discovering splice junctions with RNA-Seq. Bioinformatics. 2009;25(9):1105-1111.

45. Liao Y, Smyth GK, Shi W. featureCounts: an efficient general purpose program for assigning sequence reads to genomic features. Bioinformatics. 2014;30(7):923-930.

46. Love MI, Huber W, Anders S. Moderated estimation of fold change and dispersion for RNA-seq data with DESeq2. Genome Biol. 2014;15(12):550.

47. Subramanian A, et al. Gene set enrichment analysis: a knowledge-based approach for interpreting genome-wide expression profiles. Proc Natl Acad Sci U S A. 2005;102(43):15545-15550. 